EUROPEAN ORGANIZATION FOR NUCLEAR RESEARCH

CERN-PH-EP/2004-010

18. February 2004

\title{
Measurement of the Strange Spectral Function in Hadronic $\tau$ Decays
}

\author{
The OPAL Collaboration
}

\begin{abstract}
Tau lepton decays with open strangeness in the final state are measured with the OPAL detector at LEP to determine the strange hadronic spectral function of the $\tau$ lepton. The decays $\tau^{-} \rightarrow$ $(\mathrm{K} \pi)^{-} \nu_{\tau},(\mathrm{K} \pi \pi)^{-} \nu_{\tau}$ and $(\mathrm{K} \pi \pi \pi)^{-} \nu_{\tau}$ with final states consisting of neutral and charged kaons and pions have been studied. The invariant mass distributions of $93.4 \%$ of these final states have been experimentally determined. Monte Carlo simulations have been used for the remaining $6.6 \%$ and for the strange final states including $\eta$ mesons. The reconstructed strange final states, corrected for resolution effects and detection efficiencies, yield the strange spectral function of the $\tau$ lepton. The moments of the spectral function and the ratio of strange to non-strange moments, which are important input parameters for theoretical analyses, are determined. Furthermore, the branching fractions $B\left(\tau^{-} \rightarrow \mathrm{K}^{-} \pi^{0} \nu_{\tau}\right)=\left(0.471 \pm 0.064_{\text {stat }} \pm 0.022_{\text {sys }}\right) \%$ and $B\left(\tau^{-} \rightarrow \mathrm{K}^{-} \pi^{+} \pi^{-} \nu_{\tau}\right)=(0.415 \pm$ $\left.0.059_{\text {stat }} \pm 0.031_{\text {sys }}\right) \%$ have been measured.
\end{abstract}

( To be submitted to Euro.Phys.J. C) 
G. Abbiendi ${ }^{2}$, C. Ainsley ${ }^{5}$, P.F. Åkesson ${ }^{3, y}$, G. Alexander ${ }^{22}$, J. Allison ${ }^{16}$, P. Amaral ${ }^{9}$, G. Anagnostou ${ }^{1}$, K.J. Anderson ${ }^{9}$, S. Arcelli ${ }^{2}$, S. Asai ${ }^{23}$, D. Axen ${ }^{27}$, G. Azuelos ${ }^{18, a}$, I. Bailey ${ }^{26}$, E. Barberio $^{8, p}$, T. Barillari ${ }^{32}$, R.J. Barlow ${ }^{16}$, R.J. Batley ${ }^{5}$, P. Bechtle ${ }^{25}$, T. Behnke ${ }^{25}$, K.W. Bell ${ }^{20}$, P.J. Bell ${ }^{1}$, G. Bella ${ }^{22}$, A. Bellerive ${ }^{6}$, G. Benelli ${ }^{4}$, S. Bethke ${ }^{32}$, O. Biebel ${ }^{31}$, O. Boeriu ${ }^{10}$, P. Bock ${ }^{11}$,

M. Boutemeur ${ }^{31}$, S. Braibant ${ }^{8}$, L. Brigliadori ${ }^{2}$, R.M. Brown ${ }^{20}$, K. Buesser ${ }^{25}$, H.J. Burckhart ${ }^{8}$,

S. Campana ${ }^{4}$, R.K. Carnegie ${ }^{6}$, A.A. Carter ${ }^{13}$, J.R. Carter ${ }^{5}$, C.Y. Chang ${ }^{17}$, D.G. Charlton ${ }^{1}$,

C. Ciocca ${ }^{2}$, A. Csilling ${ }^{29}$, M. Cuffiani ${ }^{2}$, S. Dado ${ }^{21}$, A. De Roeck ${ }^{8}$, E.A. De Wolf ${ }^{8, s}$, K. Desch ${ }^{25}$, B. Dienes ${ }^{30}$, M. Donkers ${ }^{6}$, J. Dubbert ${ }^{31}$, E. Duchovni ${ }^{24}$, G. Duckeck ${ }^{31}$, I.P. Duerdoth ${ }^{16}$, E. Etzion ${ }^{22}$,

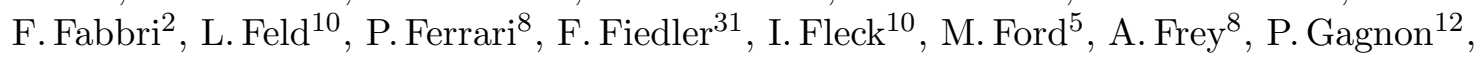
J.W. Gary ${ }^{4}$, G. Gaycken ${ }^{25}$, C. Geich-Gimbel ${ }^{3}$, G. Giacomelli ${ }^{2}$, P. Giacomelli ${ }^{2}$, M. Giunta ${ }^{4}$, J. Goldberg ${ }^{21}$, E. Gross ${ }^{24}$, J. Grunhaus ${ }^{22}$, M. Gruwé ${ }^{8}$, P.O. Günther ${ }^{3}$, A. Gupta ${ }^{9}$, C. Hajdu ${ }^{29}$, M. Hamann ${ }^{25}$, G.G.Hanson ${ }^{4}$, A. Harel ${ }^{21}$, M.Hauschild ${ }^{8}$, C.M. Hawkes ${ }^{1}$, R. Hawkings ${ }^{8}$, R.J.Hemingway ${ }^{6}$, G. Herten ${ }^{10}$, R.D. Heuer ${ }^{25}$, J.C. Hill ${ }^{5}$, K.Hoffman ${ }^{9}$, D. Horváth ${ }^{29, c}$, P. Igo-Kemenes ${ }^{11}$, K. Ishii ${ }^{23}$, H. Jeremie ${ }^{18}$, P. Jovanovic ${ }^{1}$, T.R. Junk ${ }^{6, i}$, N. Kanaya ${ }^{26}$, J. Kanzaki ${ }^{23, u}$, D. Karlen ${ }^{26}$, K. Kawagoe ${ }^{23}$, T. Kawamoto ${ }^{23}$, R.K. Keeler ${ }^{26}$, R.G. Kellogg ${ }^{17}$, B.W. Kennedy ${ }^{20}$, K. Klein ${ }^{11, t}$, A. Klier ${ }^{24}$, S. Kluth ${ }^{32}$, T. Kobayashi ${ }^{23}$, M. Kobel ${ }^{3}$, S. Komamiya ${ }^{23}$, T. Krämer ${ }^{25}$, P. Krieger ${ }^{6, l}$, J. von Krogh ${ }^{11}$, K. Kruger ${ }^{8}$, T. Kuhl ${ }^{25}$, M. Kupper ${ }^{24}$, G.D. Lafferty ${ }^{16}$, H. Landsman ${ }^{21}$, D. Lanske ${ }^{14}$, J.G. Layter ${ }^{4}$, D. Lellouch ${ }^{24}$, J. Letts ${ }^{o}$, L. Levinson ${ }^{24}$, J. Lillich ${ }^{10}$, S.L. Lloyd ${ }^{13}$, F.K. Loebinger ${ }^{16}$, J. Lu ${ }^{27, w}$, A. Ludwig ${ }^{3}$, J. Ludwig ${ }^{10}$, W. Mader ${ }^{3}$, S. Marcellini ${ }^{2}$, A.J. Martin ${ }^{13}$, G. Masetti ${ }^{2}$, T. Mashimo ${ }^{23}$, P. Mättig ${ }^{m}$, J. McKenna ${ }^{27}$, R.A. McPherson ${ }^{26}$, F. Meijers ${ }^{8}$,

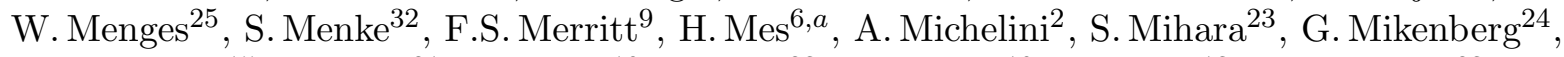
D.J.Miller ${ }^{15}$, S. Moed ${ }^{21}$, W. Mohr ${ }^{10}$, T. Mori ${ }^{23}$, A. Mutter ${ }^{10}$, K. Nagai ${ }^{13}$, I. Nakamura ${ }^{23, v}$,

H. Nanjo ${ }^{23}$, H.A. Neal ${ }^{33}$, R. Nisius ${ }^{32}$, S.W.O'Neale ${ }^{23, *}$, A. Oh ${ }^{8}$, A. Okpara ${ }^{11}$, M.J. Oreglia ${ }^{9}$, S. Orito ${ }^{23, *}$, C.Pahl ${ }^{32}$, G.Pásztor ${ }^{4, g}$, J.R. Pater ${ }^{16}$, J.E. Pilcher ${ }^{9}$, J. Pinfold ${ }^{28}$, D.E. Plane ${ }^{8}$, B. Poli ${ }^{2}$, O. Pooth ${ }^{14}$, M. Przybycień ${ }^{8, n}$, A. Quadt ${ }^{3}$, K. Rabbertz ${ }^{8, r}$, C. Rembser ${ }^{8}$, P. Renkel ${ }^{24}$, J.M. Roney ${ }^{26}$, S. Rosati ${ }^{3, y}$, Y.Rozen ${ }^{21}$, K. Runge ${ }^{10}$, K. Sachs ${ }^{6}$, T. Saeki ${ }^{23}$, E.K.G. $\operatorname{Sarkisyan}^{8, j}$, A.D. Schaile ${ }^{31}$, O. Schaile ${ }^{31}$, P. Scharff-Hansen ${ }^{8}$, J. Schieck ${ }^{32}$, T. Schörner-Sadenius ${ }^{8, a 1}$, M. Schröder ${ }^{8}$, M.Schumacher ${ }^{3}$, W.G. Scott ${ }^{20}$, R. Seuster ${ }^{14, f}$, T.G.Shears ${ }^{8, h}$, B.C.Shen ${ }^{4}$, P. Sherwood ${ }^{15}$, A.Skuja ${ }^{17}$, A.M. Smith ${ }^{8}$, R. Sobie ${ }^{26}$, S.Söldner-Rembold ${ }^{15}$, F.Spano ${ }^{9}$, A.Stahl ${ }^{3, x}$, D. Strom ${ }^{19}$, R. Ströhmer ${ }^{31}$, S. Tarem ${ }^{21}$, M. Tasevsky ${ }^{8, z}$, R. Teuscher ${ }^{9}$, M.A. Thomson ${ }^{5}$, E. Torrence ${ }^{19}$,

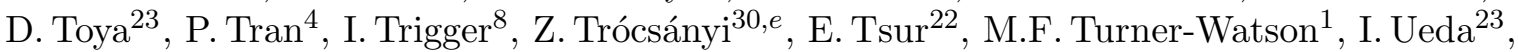
B. Ujvári ${ }^{30, e}$, C.F. Vollmer ${ }^{31}$, P. Vannerem ${ }^{10}$, R. Vértesi ${ }^{30, e}$, M. Verzocchi $^{17}$, H. $_{\text {Voss }}{ }^{8, q}$, J. Vossebeld ${ }^{8, h}$, D. Waller ${ }^{6}$, C.P. Ward ${ }^{5}$, D.R. Ward $^{5}$, P.M. Watkins ${ }^{1}$, A.T. Watson ${ }^{1}$, N.K. Watson ${ }^{1}$, P.S. Wells ${ }^{8}$, T. Wengler ${ }^{8}$, N. Wermes ${ }^{3}$, D. Wetterling ${ }^{11}$ G.W. Wilson ${ }^{16, k}$, J.A. Wilson ${ }^{1}$, G. Wolf ${ }^{24}$, T.R. Wyatt ${ }^{16}$, S. Yamashita ${ }^{23}$, D. Zer-Zion ${ }^{4}$, L. Zivkovic ${ }^{24}$

${ }^{1}$ School of Physics and Astronomy, University of Birmingham, Birmingham B15 2TT, UK

${ }^{2}$ Dipartimento di Fisica dell' Università di Bologna and INFN, I-40126 Bologna, Italy

${ }^{3}$ Physikalisches Institut, Universität Bonn, D-53115 Bonn, Germany

${ }^{4}$ Department of Physics, University of California, Riverside CA 92521, USA

${ }^{5}$ Cavendish Laboratory, Cambridge CB3 0HE, UK

${ }^{6}$ Ottawa-Carleton Institute for Physics, Department of Physics, Carleton University, Ottawa, Ontario K1S 5B6, Canada

${ }^{8}$ CERN, European Organisation for Nuclear Research, CH-1211 Geneva 23, Switzerland

${ }^{9}$ Enrico Fermi Institute and Department of Physics, University of Chicago, Chicago IL 60637, USA

${ }^{10}$ Fakultät für Physik, Albert-Ludwigs-Universität Freiburg, D-79104 Freiburg, Germany

${ }^{11}$ Physikalisches Institut, Universität Heidelberg, D-69120 Heidelberg, Germany

${ }^{12}$ Indiana University, Department of Physics, Bloomington IN 47405, USA 
${ }^{13}$ Queen Mary and Westfield College, University of London, London E1 4NS, UK

${ }^{14}$ Technische Hochschule Aachen, III Physikalisches Institut, Sommerfeldstrasse 26-28, D-52056

Aachen, Germany

${ }^{15}$ University College London, London WC1E 6BT, UK

${ }^{16}$ Department of Physics, Schuster Laboratory, The University, Manchester M13 9PL, UK

${ }^{17}$ Department of Physics, University of Maryland, College Park, MD 20742, USA

${ }^{18}$ Laboratoire de Physique Nucléaire, Université de Montréal, Montréal, Québec H3C 3J7, Canada

${ }^{19}$ University of Oregon, Department of Physics, Eugene OR 97403, USA

${ }^{20}$ CCLRC Rutherford Appleton Laboratory, Chilton, Didcot, Oxfordshire OX11 0QX, UK

${ }^{21}$ Department of Physics, Technion-Israel Institute of Technology, Haifa 32000, Israel

${ }^{22}$ Department of Physics and Astronomy, Tel Aviv University, Tel Aviv 69978, Israel

${ }^{23}$ International Centre for Elementary Particle Physics and Department of Physics, University of Tokyo, Tokyo 113-0033, and Kobe University, Kobe 657-8501, Japan

${ }^{24}$ Particle Physics Department, Weizmann Institute of Science, Rehovot 76100, Israel

${ }^{25}$ Universität Hamburg/DESY, Institut für Experimentalphysik, Notkestrasse 85, D-22607 Hamburg, Germany

${ }^{26}$ University of Victoria, Department of Physics, P O Box 3055, Victoria BC V8W 3P6, Canada

${ }^{27}$ University of British Columbia, Department of Physics, Vancouver BC V6T 1Z1, Canada

${ }^{28}$ University of Alberta, Department of Physics, Edmonton AB T6G 2J1, Canada

${ }^{29}$ Research Institute for Particle and Nuclear Physics, H-1525 Budapest, P O Box 49, Hungary

${ }^{30}$ Institute of Nuclear Research, H-4001 Debrecen, P O Box 51, Hungary

${ }^{31}$ Ludwig-Maximilians-Universität München, Sektion Physik, Am Coulombwall 1, D-85748 Garching, Germany

${ }^{32}$ Max-Planck-Institute für Physik, Föhringer Ring 6, D-80805 München, Germany

${ }^{33}$ Yale University, Department of Physics, New Haven, CT 06520, USA

${ }^{a}$ and at TRIUMF, Vancouver, Canada V6T $2 \mathrm{~A} 3$

${ }^{c}$ and Institute of Nuclear Research, Debrecen, Hungary

${ }^{e}$ and Department of Experimental Physics, University of Debrecen, Hungary

$f$ and MPI München

$g$ and Research Institute for Particle and Nuclear Physics, Budapest, Hungary

${ }^{h}$ now at University of Liverpool, Dept of Physics, Liverpool L69 3BX, U.K.

${ }^{i}$ now at Dept. Physics, University of Illinois at Urbana-Champaign, U.S.A.

$j$ and Manchester University

${ }^{k}$ now at University of Kansas, Dept of Physics and Astronomy, Lawrence, KS 66045, U.S.A.

${ }^{l}$ now at University of Toronto, Dept of Physics, Toronto, Canada

$m$ current address Bergische Universität, Wuppertal, Germany

${ }^{n}$ now at University of Mining and Metallurgy, Cracow, Poland

${ }^{o}$ now at University of California, San Diego, U.S.A.

${ }^{p}$ now at Physics Dept Southern Methodist University, Dallas, TX 75275, U.S.A.

${ }^{q}$ now at IPHE Université de Lausanne, CH-1015 Lausanne, Switzerland

${ }^{r}$ now at IEKP Universität Karlsruhe, Germany

$s$ now at Universitaire Instelling Antwerpen, Physics Department, B-2610 Antwerpen, Belgium

${ }^{t}$ now at RWTH Aachen, Germany

${ }^{u}$ and High Energy Accelerator Research Organisation (KEK), Tsukuba, Ibaraki, Japan

$v$ now at University of Pennsylvania, Philadelphia, Pennsylvania, USA

${ }^{w}$ now at TRIUMF, Vancouver, Canada

$x$ now at DESY Zeuthen

$y$ now at CERN

${ }^{z}$ now with University of Antwerp

a1 now at DESY

* Deceased 


\section{Introduction}

The $\tau$ lepton is the only lepton heavy enough to decay into hadrons. A comparison of the inclusive hadronic decay rate of the $\tau$ lepton with QCD predictions allows the measurement of some fundamental parameters of the theory. The inputs to these studies are the spectral functions that measure the transition probability to create hadronic systems of invariant mass squared $s=m^{2}$.

The energy regime accessible in $\tau$ lepton decays can be divided into two different regions: the low energy regime which has a rich resonance structure where non-perturbative QCD dominates; and the high energy regime near the kinematic limit, $s=m_{\tau}^{2}=(1.777 \mathrm{GeV})^{2}$, where perturbative QCD dominates. The high energy regime in $\tau$ lepton decays provides an environment where the strong coupling constant $\alpha_{s}$ can be measured [1-6] because the perturbative expansion converges well and non-perturbative effects are small. The measurement of the non-strange spectral function of hadronic $\tau$ lepton decays [7-9] has provided one of the most accurate measurements of $\alpha_{\mathrm{s}}$, and some very stringent tests of perturbative QCD at relatively low mass scales [10]. The spectral function of strange decays allows additional and independent tests of QCD and a measurement of the mass of the strange quark $[1,11-13]$.

The strange spectral function of the $\tau$ lepton is obtained from the invariant mass spectra of hadronic $\tau$ decay modes with net strangeness. The relevant decay channels are listed in Table 1. The only contribution to the one meson final state $\tau^{-} \rightarrow \mathrm{K}^{-} \nu_{\tau}$ is not reconstructed in this analysis. $(\mathrm{K} \pi)^{-}$ and $(\mathrm{K} \eta)^{-}$contribute to the two-meson final states. They have a total branching fraction of $1.367 \%$. The decay $\tau^{-} \rightarrow \mathrm{K}^{-} \pi^{0} \nu_{\tau}$ with a branching fraction of $0.450 \%$ can be fully reconstructed. The $\tau^{-} \rightarrow \mathrm{K}^{0} \pi^{-} \nu_{\tau}$ channel $(B=0.890 \%)$ consists of two decay chains: $\tau^{-} \rightarrow \mathrm{K}_{\mathrm{L}}^{0} \pi^{-} \nu_{\tau}(50 \%)$ and $\tau^{-} \rightarrow \mathrm{K}_{\mathrm{S}}^{0} \pi^{-} \nu_{\tau}(50 \%)$ where the $\mathrm{K}_{\mathrm{S}}^{0}$ decays to two charged (neutral) pions in roughly $68 \%(32 \%)$ of all cases. Only those final states where the $\mathrm{K}_{\mathrm{S}}^{0}$ decays to two charged pions are considered. The same applies to $\tau^{-} \rightarrow \mathrm{K}^{0} \pi^{-} \pi^{0} \nu_{\tau}(B=0.370 \%)$. This and $\tau^{-} \rightarrow \mathrm{K}^{-} \pi^{+} \pi^{-} \nu_{\tau}(B=0.280 \%)$ together form the two most important contributions to the three-meson final states. Finally, the four-meson

\footnotetext{
${ }^{1}$ In order to simplify the text we refer only to the decays of the negatively charged $\tau$ lepton. Simultaneous treatment of the charge conjugate decay is always implied.
}

\begin{tabular}{|c|c|c|c|c|c|}
\hline & \multirow[b]{2}{*}{$B_{\text {total }} / \%$} & \multicolumn{2}{|c|}{ Measured in this Paper } & \multicolumn{2}{|c|}{ Not Measured } \\
\hline & & $\tau$ decay & $B_{\mathrm{PDG}} / \%$ & $\tau$ decay & $B_{\mathrm{PDG}} / \%$ \\
\hline$(\mathrm{K})^{-}$ & $0.686 \pm 0.023$ & & & $\tau^{-} \rightarrow \mathrm{K}^{-} \nu_{\tau}$ & $0.686 \pm 0.023$ \\
\hline $\begin{array}{l}(\mathrm{K} \eta)^{-} \\
(\mathrm{K} \pi)^{-}\end{array}$ & $\begin{array}{l}0.027 \pm 0.006 \\
1.340 \pm 0.050\end{array}$ & $\begin{array}{l}\tau^{-} \rightarrow \mathrm{K}^{-} \pi^{0} \nu_{\tau} \\
\tau^{-} \rightarrow \mathrm{K}^{0} \pi^{-} \nu_{\tau}\end{array}$ & $\begin{array}{l}0.450 \pm 0.030 \\
0.890 \pm 0.040\end{array}$ & $\tau^{-} \rightarrow \mathrm{K}^{-} \eta \nu_{\tau}$ & $0.027 \pm 0.006$ \\
\hline $\begin{array}{l}(\mathrm{K} \pi \pi)^{-} \\
(\mathrm{K} \eta \pi)^{-}\end{array}$ & $\begin{array}{l}0.708 \pm 0.068 \\
0.029 \pm 0.009\end{array}$ & $\begin{array}{l}\tau^{-} \rightarrow \mathrm{K}^{0} \pi^{-} \pi^{0} \nu_{\tau} \\
\tau^{-} \rightarrow \mathrm{K}^{-} \pi^{+} \pi^{-} \nu_{\tau}\end{array}$ & $\begin{array}{l}0.370 \pm 0.040 \\
0.280 \pm 0.050\end{array}$ & $\begin{array}{l}\tau^{-} \rightarrow \mathrm{K}^{-} \pi^{0} \pi^{0} \nu_{\tau} \\
\tau^{-} \rightarrow \mathrm{K}^{*}(892) \eta \pi^{0} \nu_{\tau}\end{array}$ & $\begin{array}{l}0.058 \pm 0.023 \\
0.029 \pm 0.009\end{array}$ \\
\hline$(\mathrm{K} \pi \pi \pi)^{-}$ & $0.150 \pm 0.045$ & $\tau^{-} \rightarrow \mathrm{K}^{-} \pi^{+} \pi^{-} \pi^{0} \nu_{\tau}$ & $0.064 \pm 0.024$ & $\begin{array}{l}\tau^{-} \rightarrow \mathrm{K}^{0} \pi^{-} \pi^{0} \pi^{0} \nu_{\tau} \\
\tau^{-} \rightarrow \mathrm{K}^{-} \pi^{0} \pi^{0} \pi^{0} \nu_{\tau} \\
\tau^{-} \rightarrow \mathrm{K}^{0} \pi^{-} \pi^{+} \pi^{-} \nu_{\tau}\end{array}$ & $\begin{array}{l}0.026 \pm 0.024 \\
0.037 \pm 0.021 \\
0.023 \pm 0.020\end{array}$ \\
\hline Sum & $2.940 \pm 0.099$ & & $2.054 \pm 0.085$ & & $0.886 \pm 0.051$ \\
\hline
\end{tabular}

Table 1: Overview of all channels with net strangeness and their branching fractions [14]. 
final state $(\mathrm{K} \pi \pi \pi)^{-}$is detected via the decay $\tau^{-} \rightarrow \mathrm{K}^{-} \pi^{+} \pi^{-} \pi^{0} \nu_{\tau}$. In addition, the decay $\mathrm{K}^{-} \eta \pi^{0} \nu_{\tau}$ contributes with $0.029 \%$. Hence, $93.4 \%$ of all decay channels of the multi-meson final states with open strangeness, $(\mathrm{K} \pi)^{-},(\mathrm{K} \pi \pi)^{-}$and $(\mathrm{K} \pi \pi \pi)^{-}$, were measured. This paper describes the selection of these dominant channels and the measurement of their invariant mass spectra using data collected with the OPAL detector during the LEP-I period from 1991 to 1995 . The remaining $6.6 \%$ and the final states including $\eta$ mesons are taken from Monte Carlo simulation. The spectral function is then determined from these spectra and the spectral moments are calculated.

From an experimental point of view, one of the key issues of this analysis is the separation of charged kaons and pions via the measurement of energy loss in the OPAL jet chamber in the dense environment of multiprong $\tau$ lepton decays. Substantial improvements have been achieved compared to previous publications [15]. In particular, these improvements have made it possible to obtain a reliable $\mathrm{d} E / \mathrm{d} x$ measurement in an environment where three tracks are very close to each other. The reconstruction of neutral pions is based on the study of shower profiles in the electromagnetic calorimeter. Furthermore the identification and reconstruction of $\tau$ lepton decays with $\mathrm{K}_{\mathrm{S}}^{0} \rightarrow \pi^{+} \pi^{-}$has been achieved with high efficiency and good mass resolution.

The outline of this paper is as follows: Section 2 gives a short description of the OPAL detector concentrating on those components which are important for this analysis. In addition, the $\tau$ lepton selection and the Monte Carlo samples used are discussed. Section 3] continues with a discussion of the experimental aspects of this work. The selection of the strange hadronic $\tau$ lepton decays is described in Section 4. In Section [5] the results for the branching fractions, the strange spectral function and the spectral moments are presented together with a discussion of the systematic uncertainties. The results are summarized in Section [6]

\section{Detector and Data Samples}

\subsection{The OPAL Detector}

A detailed description of the OPAL detector can be found elsewhere [17]. A short overview is given here of those components that are vital for this analysis. Charged particles are tracked in the central detector, which is enclosed by a solenoidal magnet, providing an axial magnetic field of $0.435 \mathrm{~T}$. A high-precision silicon micro-vertex detector surrounds the beam pipe. It covers the angular region of $|\cos \theta| \leq 0.8$ and provides tracking information in the $r-\phi$ direction $^{2}$ (and $z$ from 1993) [18]. The silicon detector is surrounded by three drift chambers: a high-resolution vertex detector, a large-volume jet chamber and $z$-chambers.

The jet chamber measures the momentum and energy loss of charged particles over $98 \%$ of the solid angle. It is subdivided into 24 sectors in $r-\phi$, each containing a radial plane with 159 anode sense wires parallel to the beam pipe. Cathode wire planes form the boundaries between adjacent sectors. The 3D-coordinates of points along the trajectory of a track are determined from the sense wire position, the drift time $(r-\phi)$ and a charge division measurement $(z)$ on the sense wire. The combined momentum resolution of the OPAL tracking system is $\sigma_{p} / p^{2} \approx 1.5 \cdot 10^{-3} \mathrm{GeV}^{-1}$. From the total charge on each anode wire, the energy loss $\mathrm{d} E / \mathrm{d} x$ is calculated and used for particle identification. This measurement provides a separation between pions and kaons of at least $2 \sigma$ in the momentum range relevant for this analysis $(3 \mathrm{GeV}<p<35 \mathrm{GeV})$.

Outside the solenoid are scintillation counters which measure the time-of-flight from the interaction

\footnotetext{
${ }^{2}$ In the OPAL coordinate system the $x$-axis points to the center of the LEP ring. The $z$-axis is in the $e^{-}$beam direction. The angle $\theta$ is defined relative to the $z$-axis and $\phi$ is the azimuthal angle with respect to the $x$-axis.
} 
region and aid in the rejection of cosmic events. Next is the electromagnetic calorimeter (ECAL), which, in the barrel section, is composed of 9440 lead-glass blocks, approximately pointing to the interaction region, and covering the range $|\cos \theta|<0.82$. Each block has a $(10 \times 10) \mathrm{cm}^{2}$ profile with a depth of 24.6 radiation lengths. The resolution of the ECAL in the barrel region, including the effects of the approximately one radiation length of material in front, is $\sigma_{E} / E=$ $\sqrt{(0.16)^{2} \mathrm{GeV} / E+(0.015)^{2}}$.

The hadron calorimeter (HCAL) is beyond the electromagnetic calorimeter and is instrumented with layers of limited streamer tubes in the iron of the solenoid magnet return yoke. The outside of the hadron calorimeter is surrounded by the muon chamber system, which is composed of four layers of drift chambers in the barrel region.

\subsection{Selection of $\tau$ Lepton Candidates}

For the selection of $\tau$ lepton candidates, the standard $\tau$ selection procedure described in [16] is used. The decay of the $\mathrm{Z}^{0}$ produces a pair of back-to-back highly relativistic $\tau$ leptons. Their decay products are strongly collimated and well contained within cones of half-angle $35^{\circ}$. Therefore, each $\tau$ decay is treated separately. In order to have a precise and reliable $\mathrm{d} E / \mathrm{d} x$ measurement and to avoid regions of non-uniform calorimeter response, this analysis is restricted to the region $|\cos \theta|<0.68$. To reject background from hadronic events, exactly two cones are required and a maximum of six good ${ }^{3}$ tracks in the event is allowed. This background is further reduced by requiring that the sum of the charges of all tracks in each individual cone is \pm 1 and the net charge of the whole event is zero. A total of $162477 \tau$ cone candidates survive these selection criteria with an estimated non- $\tau$ background fraction of $1.5 \%$.

\subsection{Simulation of Events}

The $\tau$ Monte Carlo samples used consist of $200000 \tau$ pair events generated at $\sqrt{s}=m_{\mathrm{Z}^{0}}$ using KORALZ 4.02 [19] and a modified version of TAUOLA 2.4 [20]. Modifications were necessary because all four- and five-meson final states with kaons (signal as well as background) are missing in the standard version. Since the resonance structure of these channels is poorly known, only phase space distributions of these final states were generated. In addition, the resonance structure of various final states was modified to give a better description of the data [21,22]. The branching fractions of the decay channels with kaons are enhanced in this sample so that it comprises roughly a factor of ten more $\tau$ decays with kaons than expected from data. The Monte Carlo events are then reweighted to the latest branching fractions given in [14], which are used throughout the selection procedure. The Monte Carlo events were processed through the GEANT OPAL detector simulation [23].

The non- $\tau$ background was simulated using Monte Carlo samples that consist of 4000000 q $\bar{q}$ events generated with JETSET [24], 574000 Bhabha events generated with BHWIDE [25], $792000 \mu-$ pair events generated with KORALZ [19] and 1755000 two-photon events using PHOJET [26], F2GEN [27] and VERMASEREN [28, 29].

\footnotetext{
${ }^{3} \mathrm{~A}$ good track has a minimum number of 20 hits in the jet chamber, a maximum $\left|d_{0}\right|$ of $2 \mathrm{~cm}$, a maximum $\left|z_{0}\right|$ of $75 \mathrm{~cm}$, at least $100 \mathrm{MeV}$ transverse momentum and a maximum radius of the first measured point on the track of $75 \mathrm{~cm}$.
} 


\section{Identification of Hadrons in $\tau$ Final States}

\subsection{Energy Loss Measurement in $\tau$ Decays with Three or More Tracks}

A crucial part of this analysis is the identification of charged kaons via energy loss measurement in the jet chamber. Since this is the only means of distinguishing between charged pions and kaons in OPAL, a very good understanding of the effects present in the multi-track environment in $\tau$ lepton decays is vital for any analysis that requires particle identification.

The high Lorentz boost $(\gamma \approx 25)$ of the $\tau$ lepton results in its decay products being contained in a narrow cone with a typical opening angle of $5^{\circ}$. In those cases where the final state consists of more than one track, the $\mathrm{d} E / \mathrm{d} x$ measurement is known to be no longer reliable [15]. A systematic shift in the $\mathrm{d} E / \mathrm{d} x$ distribution is observed which leads to a misidentification of charged pions as kaons and thus to a reduced sensitivity in those cases where particle identification is required. The reason is explained in the following text.

When a charged particle passes through the jet chamber, it produces an ionization cloud. This cloud travels at a constant speed of $v_{D} \approx 53 \mu \mathrm{m} / \mathrm{ns}$ in the homogeneous drift field to the sense wires, where it produces a signal pulse. The pulse is integrated over a time $\Delta t=200 \mathrm{~ns}$, which corresponds to a drift distance of $\sim 1 \mathrm{~cm}$. The integral is proportional to the energy loss of the particle in this particular drift volume. The hits used for the energy loss measurement are the same as used in the track reconstruction, but they have to fulfill additional quality criteria [30]. In the following, they are called ' $\mathrm{d} E / \mathrm{d} x$ hits'.

If an additional track passes through the same sector, a second pulse will be created which overlaps with the tail of the first one (Figure (1). The contribution of the tail is determined by extrapolation of a reference pulse [30] which is normalized to the integral over the first pulse. The pulse used in all previous OPAL publications overestimates this tail contribution. The observed deviation in the energy loss measurement as a function of the distance between these two pulses $(\Delta t)$ is shown in Figure 2] This plot is obtained using tracks in $\tau$ data that have 'first hits' and 'second hits', and which have a momentum greater than $3 \mathrm{GeV}$. Such tracks arise when a second track traverses the same sector of the drift chamber and the two tracks have at least one intersection point in $r-\phi$ within the sensitive volume. Thus, for a given track, some of the hits do not need tail subtraction ('first hits') and some hits do need tail subtraction ('second hits'). Using 'first hits' only (given there are at least 20 of them) $(\mathrm{d} E / \mathrm{d} x)_{\exp }$ is calculated. The 'second hits' of the same track were
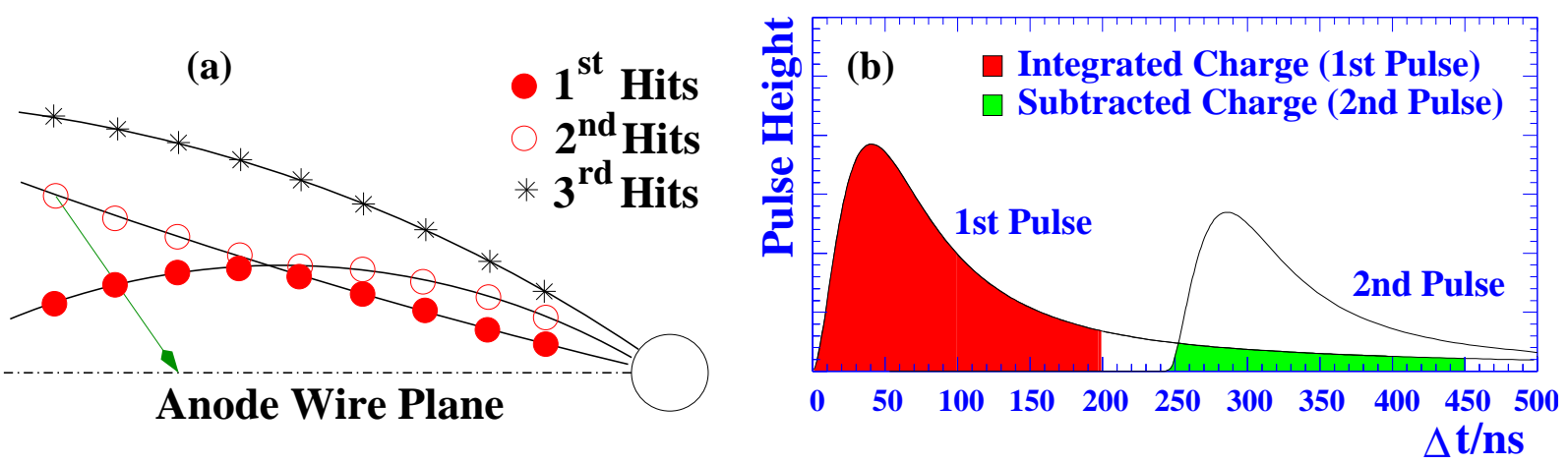

Figure 1: Illustration of the tail subtraction procedure. (a) shows one half-sector of the OPAL central drift chamber with three tracks. The arrow indicates the drift direction of the ionization cloud following a path given by a Lorentz angle of $20^{\circ}$; (b) illustrates the signal seen on the sense wire for two successive pulses. 

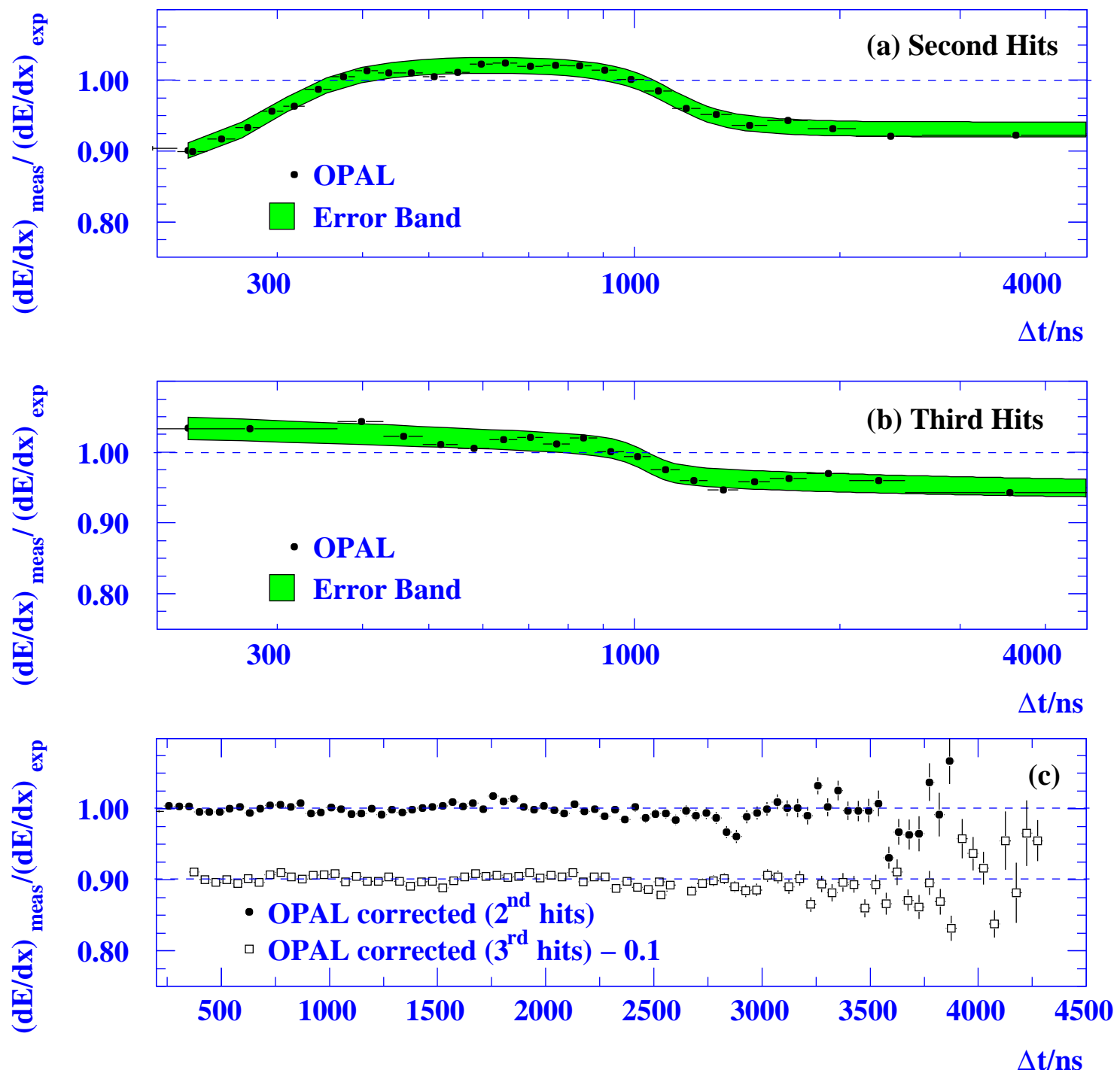

Figure 2: Measured energy loss normalized to the expectation as a function of time difference between two measured hits (200 $\mathrm{ns} \widehat{=} 1.054 \mathrm{~cm})$. The observed deviations using the standard correction are shown for (a) 'second hits' and (b) 'third hits'. The error band reflects the $1 \sigma$ error band of the parametrization. (c) shows the same distribution after all corrections have been applied. For better visibility, the distribution for 'third hits' is shifted by -0.1 in the plot.

then used to obtain $(\mathrm{d} E / \mathrm{d} x)_{\text {meas }}$ which is analyzed as a function of the drift time difference $\Delta t$ between two successive hits. The left-right ambiguity, which in principle exists if two tracks pass on either side of the anode plane, is treated properly here, since the correction is applied based on measured drift times and not on the spatial separation of the tracks.

For two hits as close as $\Delta t=200 \mathrm{~ns}$, the observed deviation is of the order of $10 \%$ of the measured $\mathrm{d} E / \mathrm{d} x$. In the region between $400 \mathrm{~ns}<\Delta t<900 \mathrm{~ns}$ the measured $\mathrm{d} E / \mathrm{d} x$ is slightly overestimated. The standard correction is finally switched off for pulses with a drift time difference of more than $1000 \mathrm{~ns}$ which produces the structure shown in Figure 2(a). The deviation as observed for "third hits' can be seen in Figure 2(b). When weighted with the $\Delta t$ distribution of all measured hits this gives on average the correct $\mathrm{d} E / \mathrm{d} x$ for high multiplicity events. In multiprong $\tau$ lepton decays this $\Delta t$ distribution differs from the average in the sense that the distribution peaks at smaller $\Delta t$. Therefore, the energy loss measurement tends to be smaller than it should be. To avoid this 
problem, previous analyses [15] exploited the $\mathrm{d} E / \mathrm{d} x$ information only of those tracks closest to the anode plane to classify the $\tau$ decay mode. This however significantly reduces the number of identified decays.

For this analysis, a new reference pulse has been developed that avoids the shortcomings of the standard one. In addition, a parametrized pulse shape was used instead of a binned one to avoid artifacts like the dip at $\Delta t \approx 500 \mathrm{~ns}$. The new reference pulse is of the form

$$
\begin{aligned}
P_{\text {norm }}= & \left(p_{1} \Delta t \exp \left(-\frac{\Delta t}{p_{2}}\right)+p_{3}(\Delta t)^{2} \exp \left(-\frac{(\Delta t)^{2}}{p_{4}}\right)\right) \\
+ & \left(p_{5} \Delta t \exp \left(-\frac{\Delta t}{p_{6}}\right)+p_{7}(\Delta t)^{2} \exp \left(-\frac{(\Delta t)^{2}}{p_{8}}\right)\right) \\
+ & \tilde{p}_{1}+\tilde{p}_{2} \Delta t
\end{aligned}
$$

with two terms to describe the short-range and the long-range part respectively plus a linear contribution. The $p_{i}$ are parameters that are optimized for the multi-track environment in $\tau$ lepton decays. The correction is applied to all hits. The effect of the correction described above can be seen in Figure 2(c) for 'second hits' and 'third hits'. Apart from this normalization correction, a further bias reduction is obtained by also correcting the shape of the reference pulse depending on the charge deposited by the preceding pulse. In general, the new reference pulse shows a steeper rise at low $\Delta t$ and a lower tail to avoid overestimation of the tail subtraction for subsequent pulses as explained above. As a result of this procedure, a $\mathrm{d} E / \mathrm{d} x$ bias reduction to $\pm 1 \%$ in $(\mathrm{d} E / \mathrm{d} x)_{\text {meas }} /(\mathrm{d} E / \mathrm{d} x)_{\exp }$ has been achieved. As the procedure is applied iteratively, i.e. the first pulse is used to correct the second, the first and corrected second pulse are used to correct a possible third pulse and so on, the chosen reference pulse is valid for any jet topology [30].

If a track is close to the anode or cathode plane in the jet chamber, the drift field is no longer homogeneous and one observes a deviation in the measured $\mathrm{d} E / \mathrm{d} x$ of the order of $3 \%$. Corrections for this effect are determined using $\mathrm{Z}^{0} \rightarrow \mu^{-} \mu^{+}$events.

When a signal is measured at a sense wire, an induced signal at the neighboring wires is also present. This effect depends on the momentum (or curvature) of the track and on $\cos \theta$. Corrections have been determined using $\mu$-pairs and $\tau \rightarrow \mu \bar{\nu}_{\mu} \nu_{\tau}$ decays. The effect is largest $(\sim 5 \%)$ for $\cos \theta \sim 0$ and large track momenta.

Finally, hits are discarded from tracks where the corresponding hit from the following or the preceding track is missing. In those cases, the measured $\mathrm{d} E / \mathrm{d} x$ is overestimated since the charge is not correctly distributed among the hits but is assigned to one hit only. By discarding this kind of hits, $(3-5) \%$ of all $\mathrm{d} E / \mathrm{d} x$ hits are lost.

The effect of all corrections can be seen in Figure 3. It shows the $\mathrm{d} E / \mathrm{d} x$ pull distribution under a pion-hypothesis for all like-sign ${ }^{4}$ tracks from 3-prong tau decays. The solid line is obtained by a fit of three Gaussians to the measured pull distribution in 9 variable momentum bins from $3 \mathrm{GeV}$ to $40 \mathrm{GeV}$. Since the number of muons in this sample is very small and their energy loss is very similar to the one for pions in the momentum range considered here, separate Gaussians for pions and muons are not needed. The free parameters in this fit are two of the three normalizations (the third one is constrained so that the sum of all three is equal to the observed number of tracks), one mean to allow for an overall shift (the position of the other two is calculated using the corresponding prediction from the Bethe-Bloch formula) and the width of the Gaussian which describes the contribution from pions. The deviation of this parameter from unity is used to

\footnotetext{
${ }^{4}$ Tracks with the same charge as the initial $\tau$ lepton.
} 


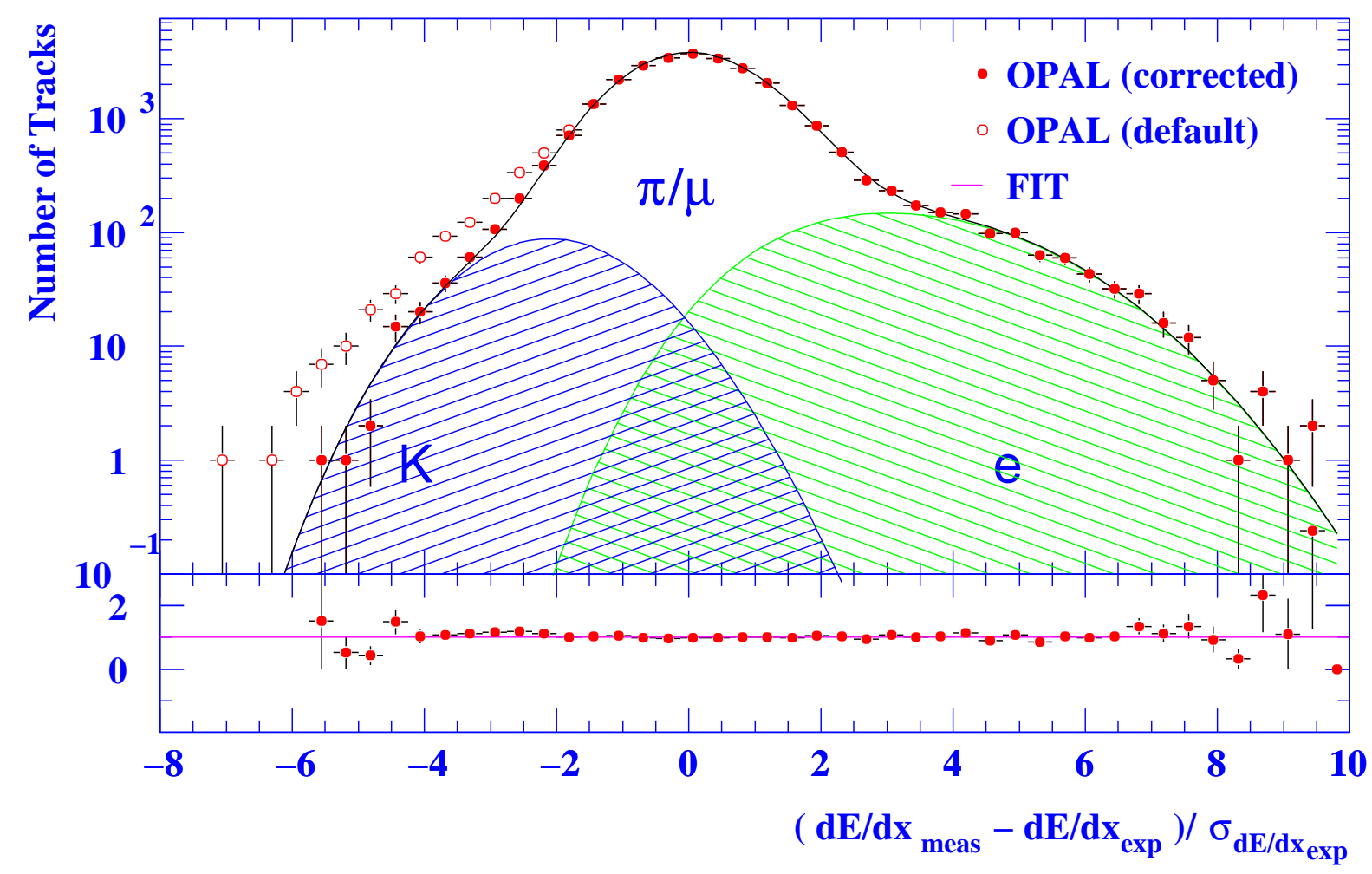

Figure 3: Pull distribution obtained under a pion hypothesis for all tracks in 3-prong $\tau$ lepton decays with a minimum momentum of $3 \mathrm{GeV}$ and a minimum number of 20 hits in the $d E / d x$ measurement. Only the tracks with the same charge as the decaying $\tau$ lepton are shown. The solid points with error bars are data after all corrections and the function shows the expectation as explained in the text. The open points in the range between - 8 to - 2 show the same distribution but without the corrections mentioned in the text. The smaller plot at the bottom shows the ratio of the full data points to the sum of the functions.

obtain correction factors for the error of the energy loss measurement. The width of the two other Gaussians is calculated assuming that the relative error is constant.

From the measured energy loss, its error and the expectation calculated using the Bethe-Bloch equation, $\chi^{2}$ probabilities are calculated that the measured energy deposition is in accordance with the expectation for a given particle type. Pion- and kaon-weights, $W_{\pi}$ and $W_{\mathrm{K}}$, as used in this paper, are then calculated by taking one minus the value of this probability. These weights acquire a sign depending on whether the actual energy loss lies above or below the expectation for a certain particle hypothesis. This means that $W_{\pi}$ is expected to be close to -1 for kaons since their energy loss per unit length is smaller in the momentum range relevant in this analysis. For electron tracks, $W_{\pi}$ is expected to be close to +1 due to the higher energy loss in this case. Whenever these quantities are used in the selection, a cut on at least $20 \mathrm{~d} E / \mathrm{d} x$ hits for this track is made implicitly.

\subsection{Photon Reconstruction and Identification of Neutral Pions}

The reconstruction of $\pi^{0}$ mesons from photon candidates starts from an algorithm that has been used in previous OPAL publications (see e.g. [7]). It is based on the analysis of shower profiles in the ECAL as a function of the energy and direction of photons. In the fit each cluster is treated 
individually. Since the lead glass blocks in the barrel have a quasi-pointing ${ }^{5}$ geometry, only the lateral shower profile is considered. This algorithm provides the number of photon candidates and their corresponding 3-vectors that best describe the measured profile of the electromagnetic cluster. All photon candidates are assumed to originate from the interaction point (approximated by the primary vertex). Since the relative branching fractions of decays with and without $\pi^{0}$ mesons are different in strange and in non-strange final states, the parameters of the algorithm were optimized for this analysis as described below.

Within each cluster only blocks with an energy of at least $150 \mathrm{MeV}$ are considered and the total cluster energy must exceed $600 \mathrm{MeV}$. The expected mean energy deposited by a minimum ionizing particle is then subtracted from all ECAL blocks hit by a charged particle before the fit. If a photon candidate is too close to the entrance point of a track into the ECAL, the track's hadronic interaction can distort the photon energy measurement. Therefore, a minimum angle of $2.8^{\circ}$ between a photon candidate and a track's ECAL entrance point is required. This value is obtained from studies of

\footnotetext{
${ }^{5}$ The longitudinal axis is pointing towards the interaction region. The blocks are tilted slightly from a perfectly pointing geometry to prevent neutral particles from escaping through the gaps between blocks.
}
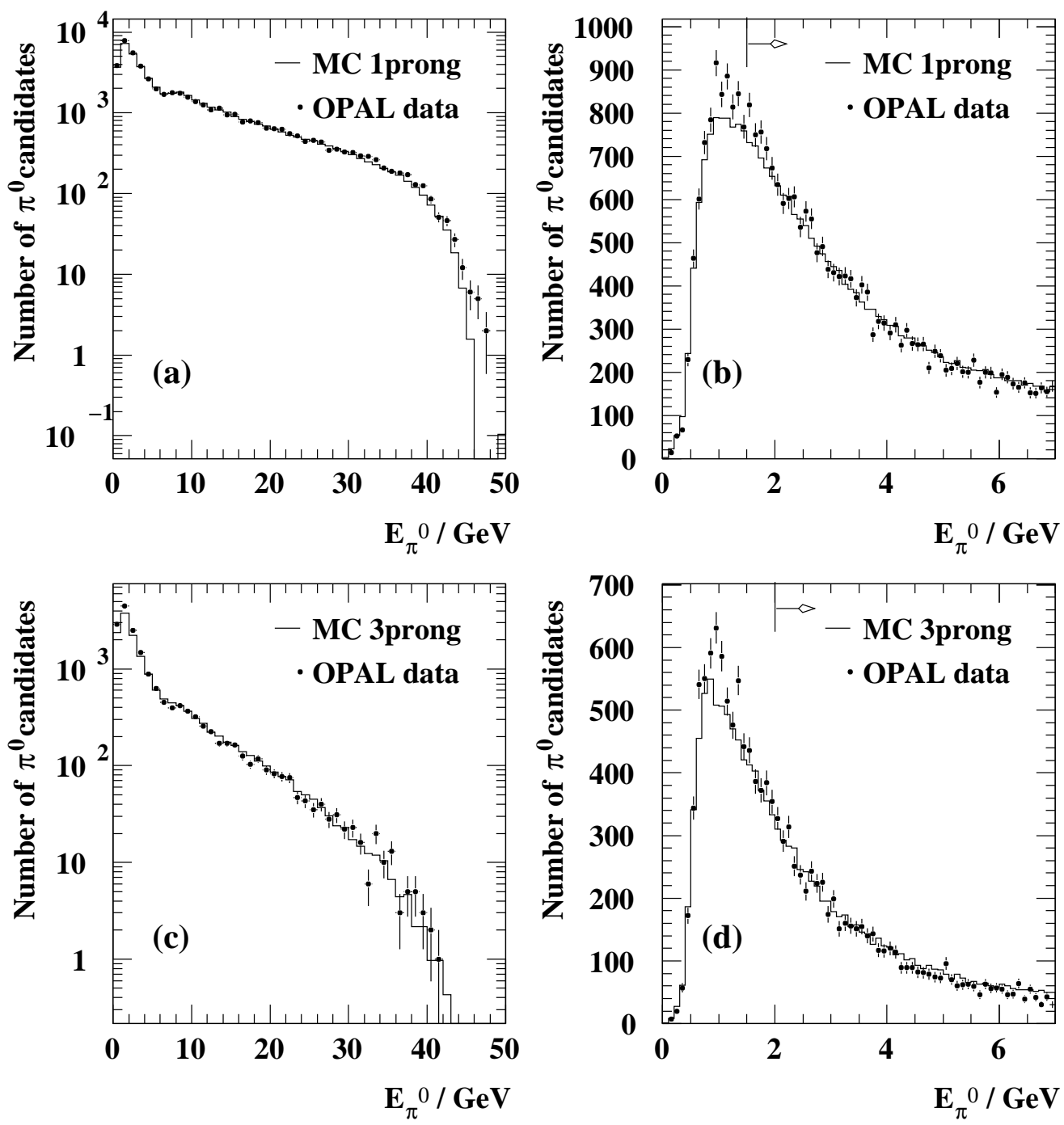

Figure 4: Energy distribution of the reconstructed $\pi^{0}$ candidates. Plots (a) and (b) show the reconstructed energy for events with one track, plots (c) and (d) for events with three tracks. All plots are normalized to the number of $\tau$ lepton decays. The arrows indicate the region selected. 
the rate of fake $\pi^{0}$ mesons in the decay $\tau^{-} \rightarrow \mathrm{K}^{-} \nu_{\tau}$ and subsequent optimization. To improve the energy resolution and the purity of the selection, a pairing algorithm is applied to recombine fake photon candidates with the closest photon candidates that were wrongly split up by the reconstruction procedure. The recombination is performed using the Jade jet finding scheme with the P0 option [31]. Jet resolution parameter $y_{\text {cut }}$ values are optimized to obtain the best description of the number of expected photons using Monte Carlo events. Two $y_{\text {cut }}$ values are determined, one for clusters where a track is pointing to one of the blocks in the cluster and one for those without tracks. This is necessary since the hadronic interaction of charged particles disturbs the shower profile. The optimized $y_{\text {cut }}$ values are -3 and -4.6 for clusters with and without tracks, respectively. The angular resolution of this algorithm is $2.1^{\circ}$ for clusters with tracks and $1.7^{\circ}$ for clusters without tracks. Since these opening angles correspond directly to the energy of the neutral pion, photon candidates with an energy of more than $7.5 \mathrm{GeV}$ are directly interpreted as neutral pion and the 4 -vector is corrected to account for the $\pi^{0}$ mass. The energy of the reconstructed $\pi^{0}$ candidates for events with one and three tracks is shown in Figure 4. The plots are normalized to the number of $\tau$ decays in the event sample. Neutral pion candidates with an energy below $1.5 \mathrm{GeV}$ in the 1-prong case and $2 \mathrm{GeV}$ in the 3-prong case are rejected.

For the remaining photon candidates, all two-photon combinations are tested. The combination which results in the maximum number of neutral pions with invariant two-photon masses not exceeding the $\pi^{0}$ mass by more than $1.5 \sigma$ is retained. A fit with a $\pi^{0}$ mass constraint is then applied to all $\pi^{0}$ candidates. All $\pi^{0}$ candidates are assumed to originate from the primary interaction point.

\subsection{Identification of $\mathrm{K}_{\mathrm{S}}^{0}$}

The $\mathrm{K}^{0}$ signal consists of $50 \% \mathrm{~K}_{\mathrm{L}}^{0}$ and $50 \% \mathrm{~K}_{\mathrm{S}}^{0}$. The signature of a $\mathrm{K}_{\mathrm{L}}^{0}$ decay is a large energy deposit in the hadron calorimeter without an associated track pointing to the cluster. The resolution of the OPAL hadron calorimeter would not allow for a clean reconstruction of this channel, thus it is not considered here. For the $\mathrm{K}_{\mathrm{S}}^{0}$, two decay modes are dominant, $\mathrm{K}_{\mathrm{S}}^{0} \rightarrow \pi^{0} \pi^{0}(\approx 32 \%)$ and $\mathrm{K}_{\mathrm{S}}^{0} \rightarrow \pi^{+} \pi^{-}$ $(\approx 68 \%)$. In this analysis only the latter is considered since the photon reconstruction algorithm exploits the quasi-pointing geometry of the electromagnetic calorimeter in the barrel (see Section 3.2). Thus only photons from the primary vertex can be properly reconstructed.

The selection starts by combining each pair of oppositely charged tracks. Each track must have a transverse momentum with respect to the beam axis of $p_{\mathrm{T}} \geq 150 \mathrm{MeV}$, a minimum of 20 out of 159 possible hits in CJ, at least $20 \%$ of all geometrically possible hits and a maximum $\chi^{2}$ for the track fit of 50. For each combination of tracks, their intersection points in the plane perpendicular to the beam axis are calculated. The one with a radius less than $150 \mathrm{~cm}$ is selected as the secondary vertex. If two vertices are found that satisfy this condition, the one with the first measured hit closest to the intersection point is selected. In addition, the $z$-coordinate of the vertex has to satisfy $\left|z_{\mathrm{V}}\right|<80 \mathrm{~cm}$.

The variables used in the selection described below are shown in Figure 5 For each $\mathrm{K}_{\mathrm{S}}^{0}$ candidate, the angle between the reconstructed momentum of the candidate vertex and the $\mathrm{K}_{\mathrm{S}}^{0}$ direction of flight must be less than $\left|\Theta_{\mathrm{V}}\right|<0.5^{\circ}$. If the number of $\mathrm{d} E / \mathrm{d} x$-hits is more than 20 on at least one of the tracks, the energy loss information is used to veto photon conversions. All candidates where at least one track has a pion weight of more than 0.98 are rejected. To further reduce the background from photon conversions, the invariant mass of the $\mathrm{K}_{\mathrm{S}}^{0}$ candidate is calculated using electron hypothesis for both tracks. All combinations with a mass $m_{\mathrm{ee}}<0.1 \mathrm{GeV}$ are rejected. The remaining background that mainly comes from 3-prong $\tau$ lepton decays or from wrong track combinations is rejected by applying cuts on the impact parameters (with respect to the nominal interaction point $\left.d_{0}\right)$ of the two tracks. The $d_{0}$ values must have opposite sign according to the OPAL convention and the absolute values have to satisfy $\min \left(\left|d_{0}^{(1)}\right|,\left|d_{0}^{(2)}\right|\right)>0.03 \mathrm{~cm}$ and $\max \left(\left|d_{0}^{(1)}\right|,\left|d_{0}^{(2)}\right|\right)>0.1 \mathrm{~cm}$. 

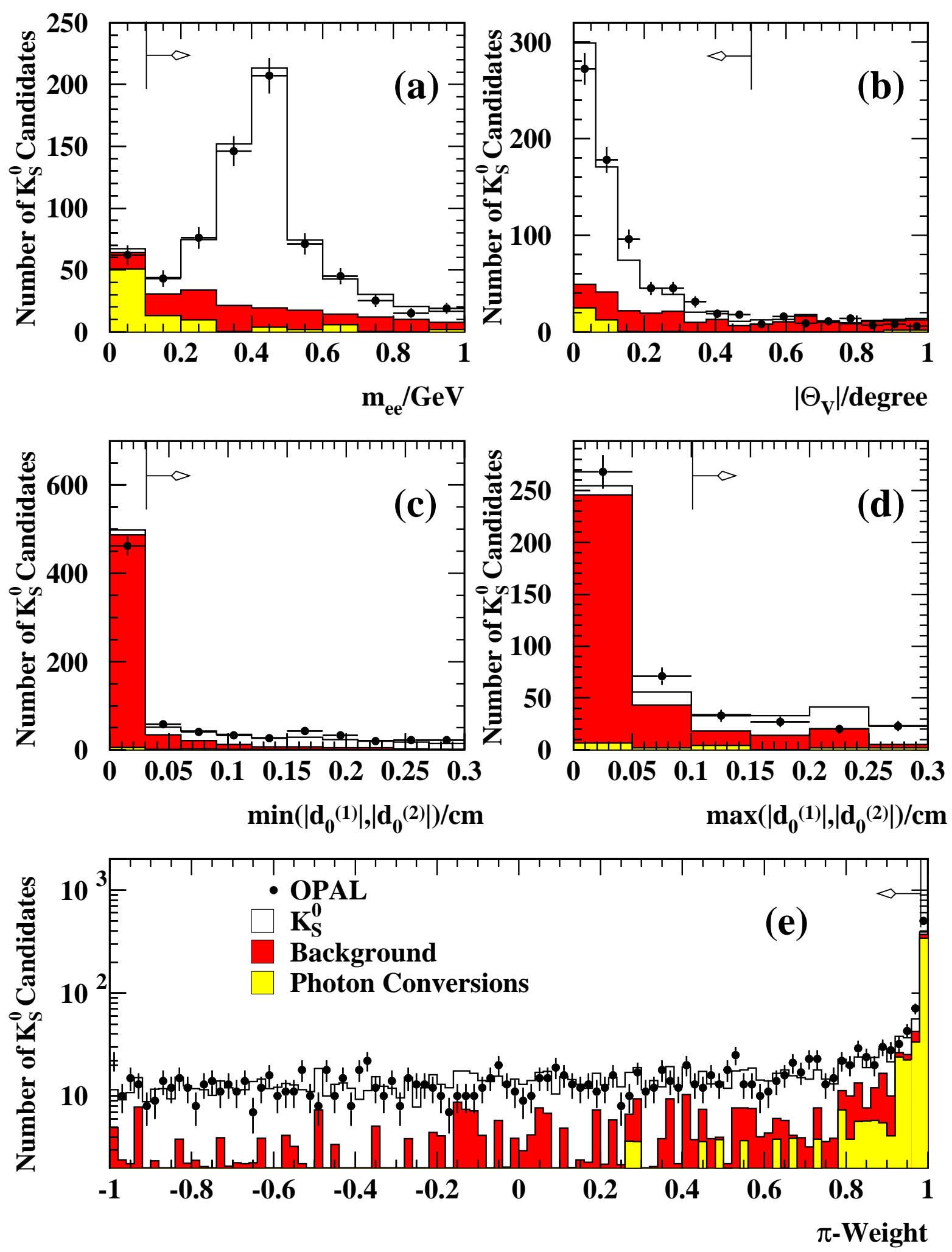

Figure 5: Variables used in the $\mathrm{K}_{\mathrm{S}}^{0}$ selection. A detailed description of all variables is given in the text. The dots represent the data and the open histogram is Monte Carlo signal. The shaded areas show the background where photon conversions are marked separately. The arrows indicate the region selected. For all plots, all selection cuts have been applied except for the cut on the variable shown. All plots are normalized to the number of $\tau$ decays. 

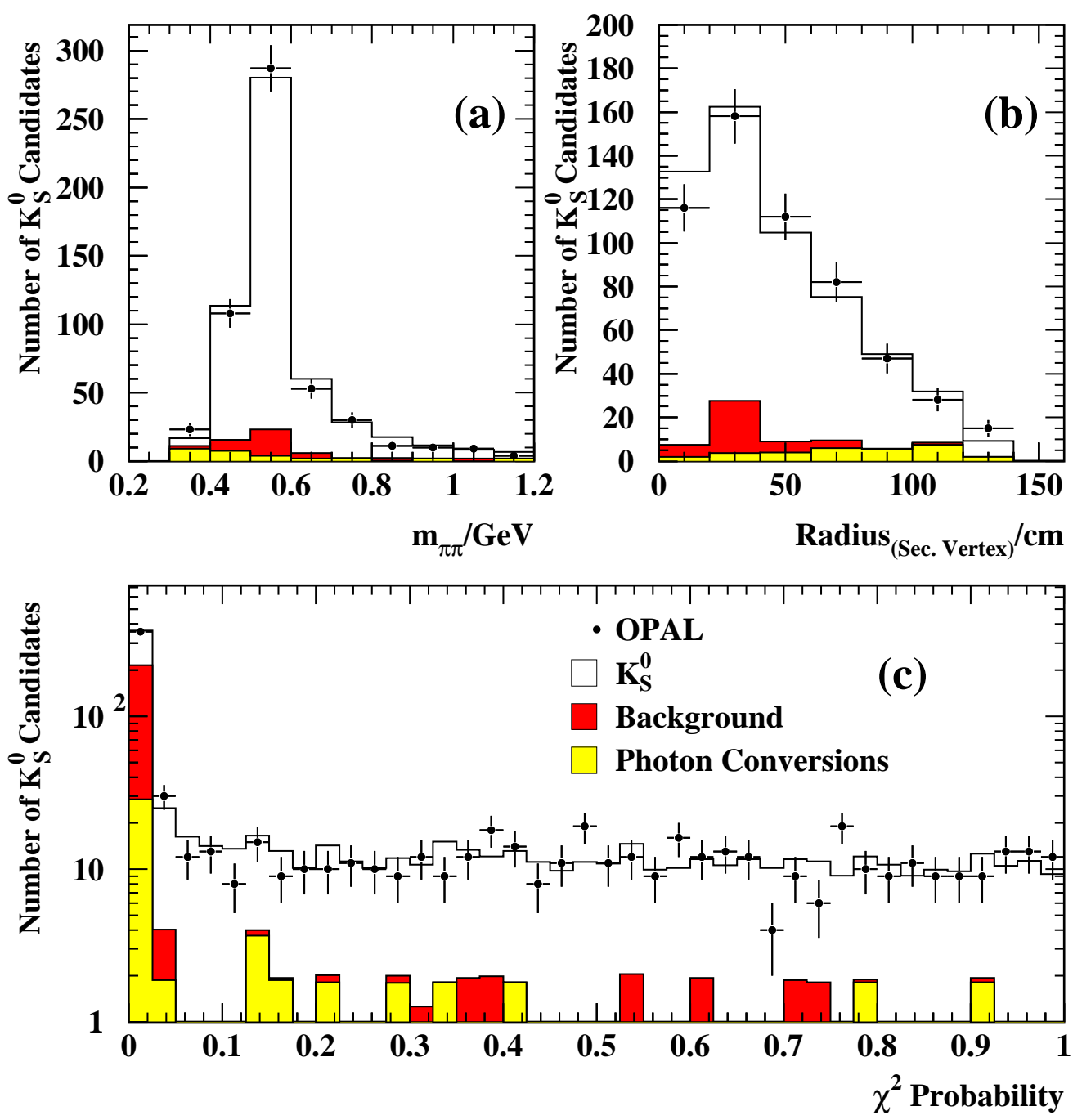

Figure 6: Result of the $\mathrm{K}_{\mathrm{S}}^{0}$ selection. Plot (a) shows the invariant mass distribution of the $\mathrm{K}_{\mathrm{S}}^{0}$ candidates under pion hypothesis before the kinematic fit. Plot (b) shows the radius of the reconstructed secondary vertex and plot (c) the distribution of the $\chi^{2}$-probability of the 2C-fit. A cut is applied on the probability at $10^{-5}$. The dots represent the data and the open histogram is the Monte Carlo signal. The shaded areas show the background where photon conversions are marked separately. All plots are normalized to the number of $\tau$ decays.

The remaining $\mathrm{K}_{\mathrm{S}}^{0}$ candidates must have a momentum of $p_{\mathrm{K}_{\mathrm{S}}^{0}}>3 \mathrm{GeV}$. A $3 \mathrm{D}$ vertex fit is applied to each candidate, that includes a constraint of the invariant two-track mass under the pion hypothesis to the nominal $\mathrm{K}_{\mathrm{S}}^{0}$ mass. This is a $2 \mathrm{C}$ fit and a cut on the $\chi^{2}$ probability at $10^{-5}$ is applied. The invariant two-track mass under the pion hypothesis before the kinematic fit can be found in Figure [6] together with the radius of the reconstructed secondary vertex and the $\chi^{2}$ probability of the kinematic fit. In Figure 6(a) the $\pi \pi$-invariant mass spectrum is shown without any vertex constraint. If more than one $\mathrm{K}_{\mathrm{S}}^{0}$ candidate shares the same track, the one with the smallest deviation from the nominal $\mathrm{K}_{\mathrm{S}}^{0}$ mass before the fit is selected.

After this selection procedure, a total of $535 \mathrm{~K}_{\mathrm{S}}^{0}$ candidates remain with an estimated purity of $82 \%$. About $70 \%$ of the background consists of wrong combinations of tracks, and $30 \%$ comes from photon conversions. In one data event, two $\mathrm{K}_{\mathrm{S}}^{0}$ candidates are found within one cone. This event is considered to be background. 


\section{Identification of $\tau$ Final States}

For the selection of the various final states, a cut-based procedure is used where each $\tau$ decay is treated independently. For all selected decay modes, the cone axis, calculated from the momenta of all tracks and neutral clusters identified in the electromagnetic calorimeter, must have a polar angle within $|\cos \theta|<0.68$ for the reasons explained above. Each selected cone must have at least one good track coming from the interaction point and the summed momenta of all tracks have to be less than the beam energy. Since there is at least one hadron in the final states considered here, the total energy deposited in the hadron calorimeter within the cone is required to exceed $1 \mathrm{GeV}$.

\subsection{Corrections to the Invariant Mass Spectra}

The spectral function from $\tau$ lepton decays is a weighted invariant mass distribution of all hadronic final states with strangeness. The various final states have different experimental resolutions and migration effects that must be corrected for. To correct the observed data, the following method was used. The elements $c_{i j}$ of the inverse detector response matrix were determined directly from Monte Carlo. They represent the probability that an event reconstructed in bin $j$ was generated in bin $i$. To calculate this probability Monte Carlo samples for the signal channels with mass distributions according to phase space were used. The corrected distribution was then obtained by

$$
g_{i}^{\text {Corrected }}=\sum_{j} c_{i j}\left(g_{j}^{\text {DATA }}-g_{j}^{\text {Background }}\right)
$$

where $g_{j}^{\text {DATA }}$ is the number of events in the data with a reconstructed mass in bin $j, g_{j}^{\text {Background }}$ is the number of background events predicted in bin $j$ and $g_{i}^{\text {Corrected }}$ the number of events in mass bin $i$ after correction.

The corrected distributions will in general be biased towards the Monte Carlo input distributions. To reduce the bias from this approach, the method was applied iteratively. The result of the preceding iteration was used to refine the elements of the inverse detector matrix. The optimal number of iterations was determined, using Monte Carlo simulations, to be two for all final states considered here. The corresponding systematic uncertainties are discussed in Section 5.4. Finally, detection efficiency corrections were applied to the corrected invariant mass distributions. The bin width of $150 \mathrm{MeV}$ of the measured invariant mass spectra were chosen according to the mass resolution of the $\tau$ decay channels measured.

\section{$4.2 \quad(\mathrm{~K} \pi)^{-}$Final States}

The $(\mathrm{K} \pi)^{-}$mass spectrum consists of two measured modes, $\mathrm{K}^{-} \pi^{0} \nu_{\tau}$ and $\mathrm{K}^{0} \pi^{-} \nu_{\tau}$. From the latter decay mode, only the decays $\mathrm{K}_{\mathrm{S}}^{0} \rightarrow \pi^{+} \pi^{-}$are measured. The $\mathrm{K}^{*}(892)$ dominance is well established in both cases.

\subsection{1 $\quad \mathrm{K}^{-} \pi^{0} \nu_{\tau}$}

In the $\mathrm{K}^{-} \pi^{0} \nu_{\tau}$ selection, exactly one good track coming from the primary vertex is required. This track must have a minimum momentum of $p>3 \mathrm{GeV}$. For the track to be selected as a kaon, the pion weight has to satisfy $W_{\pi}<-0.98$ and the kaon weight $W_{\mathrm{K}}<0.6$. Furthermore, exactly one 
identified neutral pion is required with $E_{\min }^{\pi^{0}}>1.5 \mathrm{GeV}$ selected using the algorithm explained in Section 3.2 The variables used in this selection are shown in Figures 8 and 17

From this selection 360 events are seen in the data with 190.5 background events predicted from Monte Carlo. This corresponds to a background fraction of $54 \%$. The invariant mass spectrum can be seen in Figure 9. The mass resolution in this channel is approximately $40 \mathrm{MeV}$. The main background comes from $\tau^{-} \rightarrow \mathrm{K}^{-} \nu_{\tau}$ decays where one fake neutral pion was reconstructed. Additional sources of background are $\tau^{-} \rightarrow \pi^{-} \pi^{0} \nu_{\tau}, \tau^{-} \rightarrow \mathrm{K}^{-} \mathrm{K}^{0} \nu_{\tau}$ and $\tau^{-} \rightarrow \mathrm{K}^{0} \mathrm{~K}^{-} \pi^{0} \nu_{\tau}$ where the $\mathrm{K}^{0}$ is a $\mathrm{K}_{\mathrm{S}}^{0}$ decaying to two neutral pions or a $\mathrm{K}_{\mathrm{L}}^{0}$ which does not decay within the jet chamber.

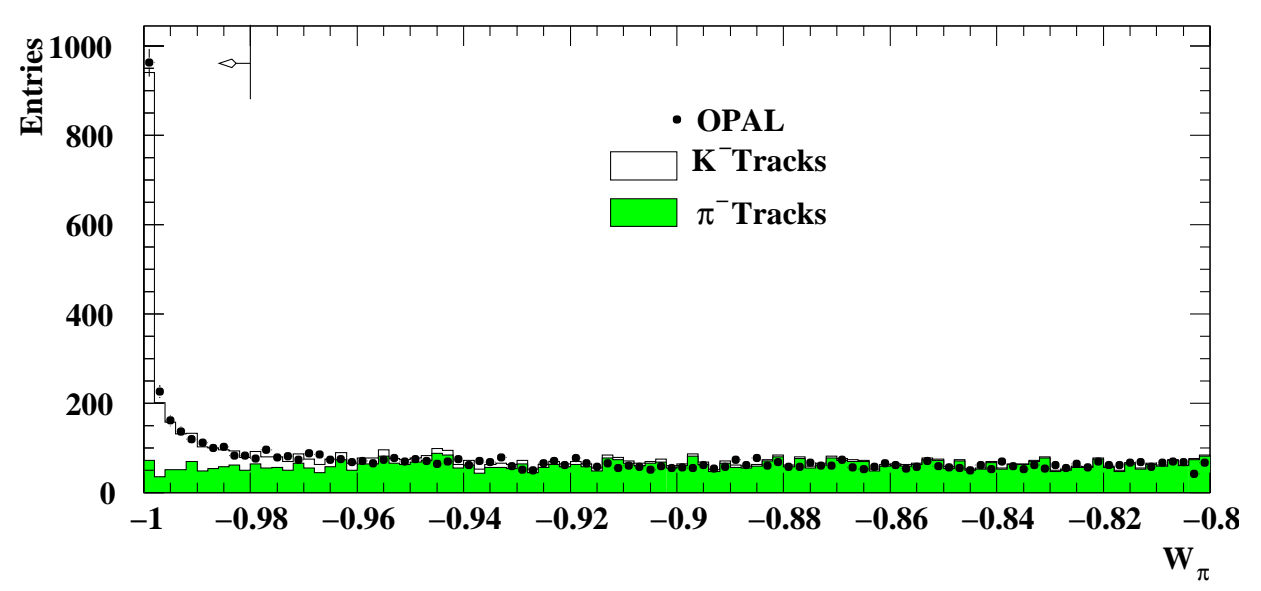

Figure 7: The pion weight $W_{\pi}$ for 1-prong $\tau$ decays in the range $(-1:-0.8)$. The dots are the data points. The open (shaded) histogram denotes the contribution from kaon (pion) tracks as predicted by the Monte Carlo simulation. The plot is normalized to the number of $\tau$ decays. Events on the side of the direction of the arrow are considered kaon candidates.
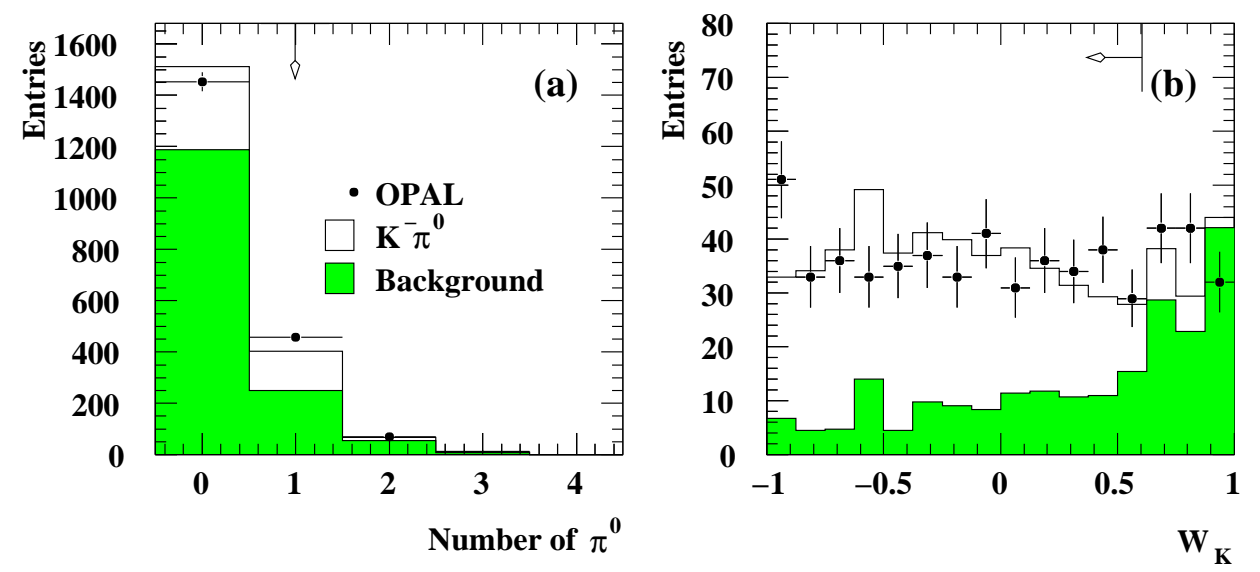

Figure 8: Variables used in the $\mathrm{K}^{-} \pi^{0} \nu_{\tau}$ selection. The dots are the data points and the open histogram is the prediction from the Monte Carlo. Plot (a) shows the number of reconstructed $\pi^{0}$ mesons with $E>1.5 \mathrm{GeV}$. In this plot the shaded area is the background prediction from the Monte Carlo. Plot (b) shows the kaon weight as explained in the text. Here the shaded area represents the expected background from pion tracks. The arrows indicate the events kept in the selection. For both plots, all selection cuts have been applied except for the cut on the variable shown. 

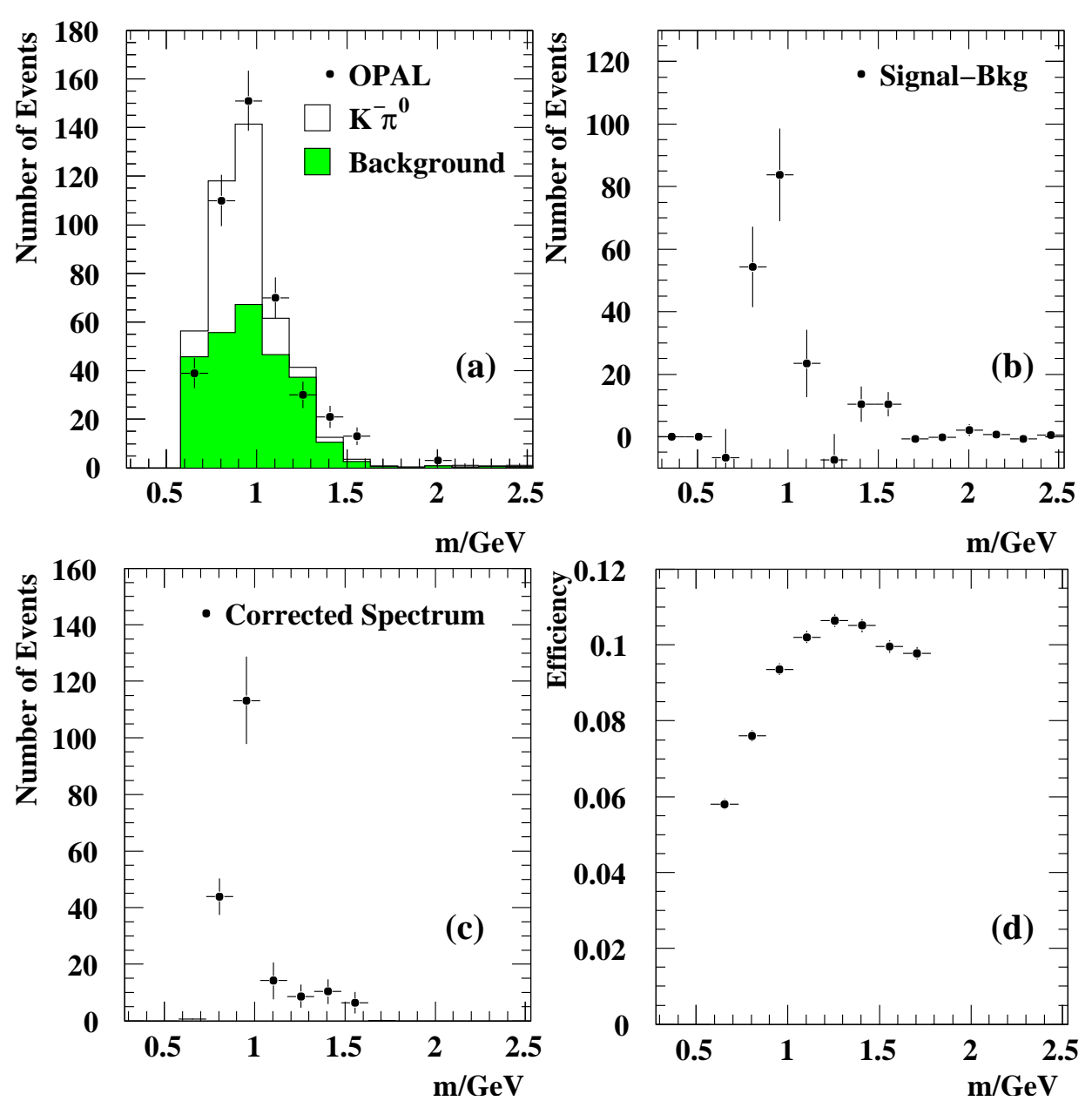

Figure 9: Result of the $\mathrm{K}^{-} \pi^{0} \nu_{\tau}$ selection. Plot (a) shows the measured invariant mass spectrum. The dots are the data, the open histogram is the Monte Carlo signal and the shaded area is the background. Plot (b) shows the background subtracted spectrum, (c) shows the corrected spectrum. Note that the correlation between neighboring bins in the corrected spectrum is of the order of $50 \%$. Plot (d) is the selection efficiency as a function of the invariant mass.

\subsection{2 $\quad \mathrm{K}^{0} \pi^{-} \nu_{\tau}$}

The selection is very similar to that for the $\mathrm{K}^{-} \pi^{0} \nu_{\tau}$ final state. Here exactly one identified $\mathrm{K}_{\mathrm{S}}^{0}$ is required using the procedure from Section 3.3. In addition, one good track from the primary vertex is required. If the momentum of this track lies above the kinematically allowed minimum for a kaon, the same identification procedure as mentioned above is applied to veto decays $\tau^{-} \rightarrow \mathrm{K}^{-} \mathrm{K}^{0} \nu_{\tau}$. Only events with zero reconstructed $\pi^{0}$ mesons are accepted. The variables used in the selection are shown in Figure 10.

From this selection 361 events are expected with a background fraction of $47 \%$, and 344 are seen in the data. The main background contributions come from decays $\tau^{-} \rightarrow \mathrm{K}^{0} \overline{\mathrm{K}}^{0} \pi^{-} \nu_{\tau}, \tau^{-} \rightarrow \mathrm{K}^{-} \mathrm{K}^{0} \nu_{\tau}$ and $\tau^{-} \rightarrow \mathrm{K}^{0} \pi^{-} \pi^{0} \nu_{\tau}$ where the neutral pion escapes detection. The invariant mass spectrum for this channel is shown in Figure 11. The mass resolution in this channel is approximately $60 \mathrm{MeV}$. 

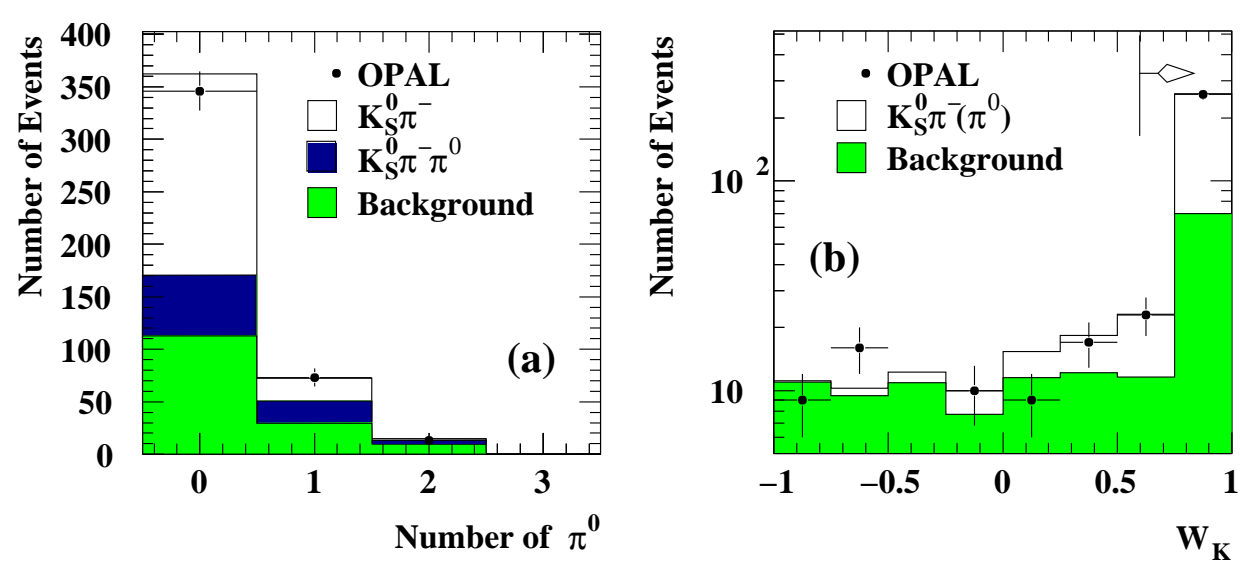

Figure 10: Variables used in the $\mathrm{K}^{0} \pi^{-} \nu_{\tau}$ and $\mathrm{K}^{0} \pi^{-} \pi^{0} \nu_{\tau}$ selection. (a) shows the number of reconstructed $\pi^{0}$ mesons. The dots are the data, the open histogram is the signal. The dark-shaded area represents the contribution from $\mathrm{K}^{0} \pi^{-} \pi^{0} \nu_{\tau}$ final states, the light-shaded area denotes other background channels. (b) is the kaon weight of the primary track
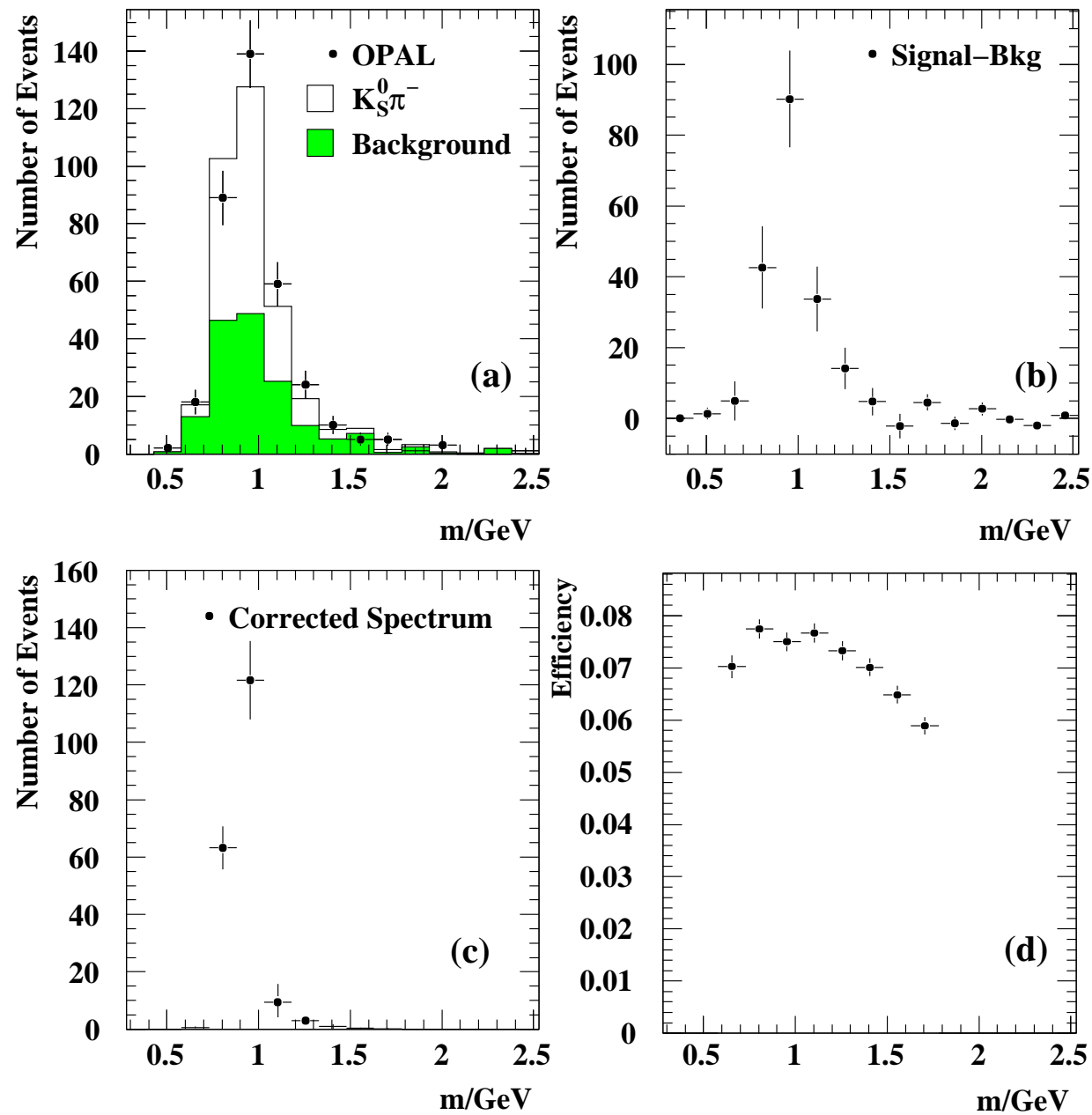

Figure 11: Result of the $\mathrm{K}^{0} \pi^{-} \nu_{\tau}$ selection. Plot (a) shows the measured invariant mass spectrum. The dots are the data, the open histogram is the Monte Carlo signal and the shaded area is the background. Plot (b) shows the background subtracted spectrum, (c) the corrected spectrum. Note that the correlation between neighboring bins in the corrected spectrum is of the order of $50 \%$. Plot (d) is the selection efficiency as a function of the invariant mass. 


\section{$4.3(\mathrm{~K} \pi \pi)^{-}$Final States}

The $(\mathrm{K} \pi \pi)^{-}$final state consists of the decay modes: $\mathrm{K}^{-} \pi^{+} \pi^{-} \nu_{\tau}, \mathrm{K}^{0} \pi^{-} \pi^{0} \nu_{\tau}$ and $\mathrm{K}^{-} \pi^{0} \pi^{0} \nu_{\tau}$. To select these final states, the following procedure is applied.

\subsection{1 $\mathrm{K}^{-} \pi^{+} \pi^{-} \nu_{\tau}$}

The selection starts by requiring exactly three good tracks coming from the interaction point. These tracks are fitted to a common vertex and the fit probability is required to be larger than $10^{-7}$. In addition, each pair of oppositely charged tracks has to fail the selection criteria for neutral kaons as defined in Section 3.3. These two requirements reduce the background from photon conversions and decays containing $\mathrm{K}_{\mathrm{S}}^{0}$.

To identify the kaon in the decay, one of the like-sign candidate tracks must have $p>3 \mathrm{GeV}$ and $W_{\pi}<-0.9$. To further reduce the pion background among these candidate tracks, $W_{\mathrm{K}}$ and $W_{\pi}$ are input to a neural network. The track is rejected if the output of the neural network is below 0.3 (see Figure 12(c)). Exactly one like-sign track is allowed to fulfill these requirements, otherwise the decay is treated as background. If the momentum of the unlike-sign track is consistent with the $\tau^{-} \rightarrow \pi^{-} \mathrm{K}^{-} \mathrm{K}^{+} \nu_{\tau}$ hypothesis, this $\tau$ decay is only accepted if $W_{\pi}>-0.95$ (see Figure 12(d)).

The algorithm for identifying neutral pions (Section 3.2) is then applied to the selected cones. For this channel, the number of reconstructed $\pi^{0}$ mesons with an energy greater than $2 \mathrm{GeV}$ is required to be zero (see Figure 12(b)). Otherwise this $\tau$ decay is treated as background. To further improve the purity of the selection, the cosine of the decay angle in the rest frame of the $\tau$ lepton, the socalled Gottfried-Jackson angle $\Theta^{*}$ is calculated. The $\cos \Theta^{*}$ distribution is shown in Figure 12(a). For events where the kaon hypothesis was applied to the wrong track or the number of identified neutral pions does not correspond to the true number, this calculation leads to unphysical values of that variable. Due to resolution effects, correctly identified signal events can also give values beyond \pm 1 . Therefore, a cut was applied at $\cos \Theta^{*}= \pm 1.2$. The contribution from $\tau^{-} \rightarrow \mathrm{K}^{-} \pi^{+} \pi^{-}\left(\mathrm{n} \pi^{0}\right) \nu_{\tau}$ events is included in the background estimate.

From this selection 269 events are seen in the data with a contribution of 149.8 background events predicted from Monte Carlo. This corresponds to a background fraction of $63 \%$. The main background contribution comes from decays $\tau^{-} \rightarrow \pi^{-} \pi^{-} \pi^{+} \nu_{\tau}, \tau^{-} \rightarrow \pi^{-} \mathrm{K}^{-} \mathrm{K}^{+} \nu_{\tau}$ and $\tau^{-} \rightarrow \mathrm{K}^{-} \pi^{+} \pi^{-} \pi^{0} \nu_{\tau}$, where the $\pi^{0}$ meson escapes detection. The invariant mass spectrum can be found in Figure 13. The mass resolution in this channel is approximately $20 \mathrm{MeV}$.

\subsection{2 $\quad \mathrm{K}^{0} \pi^{-} \pi^{0} \nu_{\tau}$}

Exactly one identified $\mathrm{K}_{\mathrm{S}}^{0}$ and exactly one $\pi^{0}$ meson with an energy $E^{\pi^{0}}>2 \mathrm{GeV}$ is required for this final state. The pion candidate track has to satisfy the same requirements as for $(\mathrm{K} \pi)^{-}$final states.

From this selection, 65 events are expected and 67 are seen in the data with a background fraction of $72 \%$. The main background contribution comes from decays $\tau^{-} \rightarrow \mathrm{K}^{0} \pi^{-} \nu_{\tau}$ where the neutral pion escapes detection. The invariant mass spectrum can be seen in Figure 14, The mass resolution in this channel is approximately $100 \mathrm{MeV}$. 

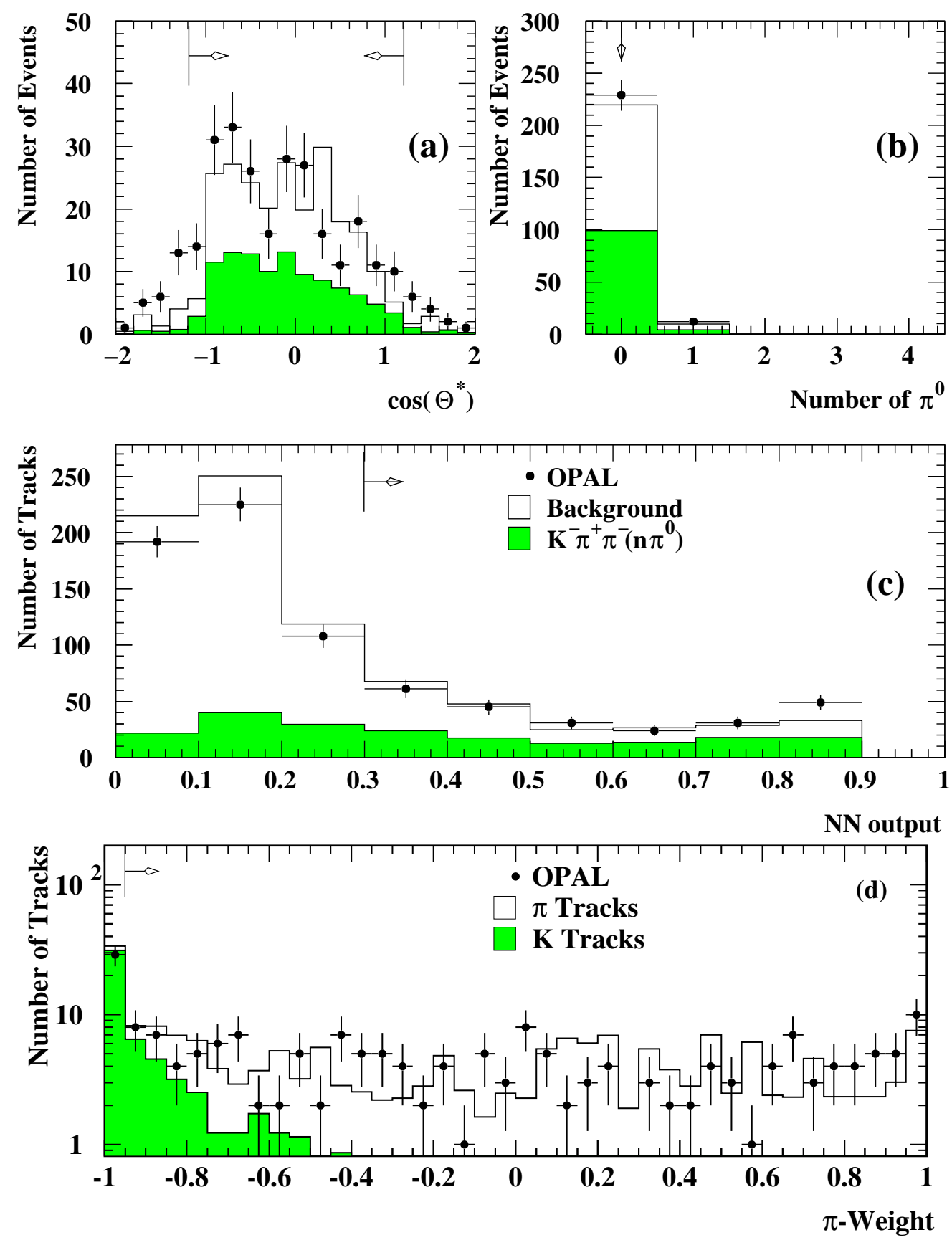

Figure 12: Variables used in the $\mathrm{K}^{-} \pi^{+} \pi^{-} \nu_{\tau}$ selection. Plot (a) shows the cosine of the GottfriedJackson angle $\Theta^{*}$, (b) the number of reconstructed neutral pions and (c) the output of the neural network. The dots are the data, the open histogram is the background prediction from the Monte Carlo and the shaded histogram denotes the contribution from $\mathrm{K}^{-} \pi^{+} \pi^{-} n$ $\pi^{0} \nu_{\tau}$ final states. (d) shows the pion weight of the unlike-sign track in the $\mathrm{K}^{-} \pi^{+} \pi^{-} \nu_{\tau}$ selection. Here the open histogram denotes the contribution from pion tracks, the shaded histogram is the contribution from kaon tracks. The dots are the data. For all plots, all selection cuts have been applied except for the cut on the variable shown. The arrows indicate the region selected. 

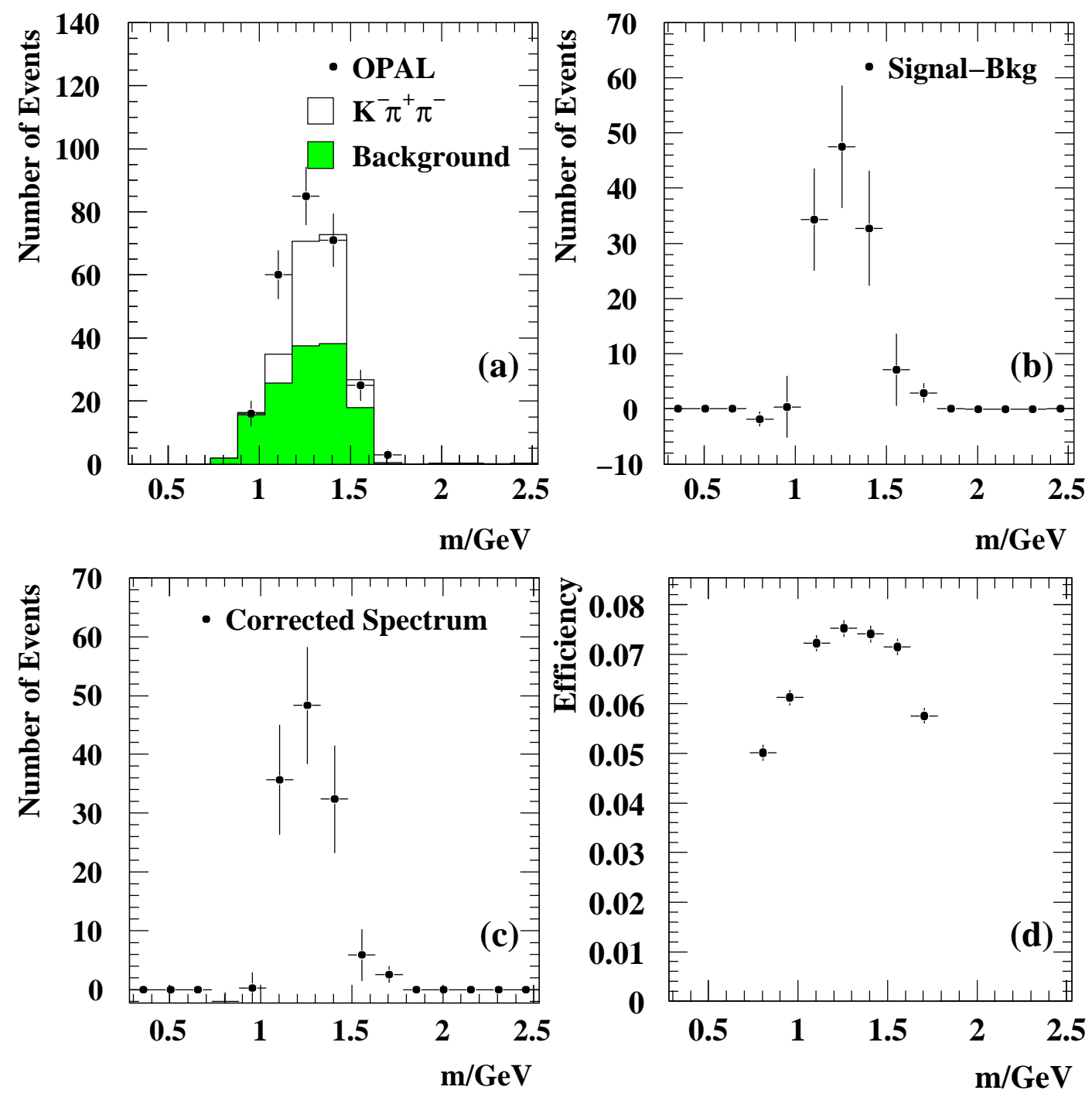

Figure 13: Result of the $\mathrm{K}^{-} \pi^{+} \pi^{-} \nu_{\tau}$ selection. Plot (a) shows the measured invariant mass spectrum. The dots are the data, the open histogram is the Monte Carlo signal and the shaded area is the background. Plot (b) shows the background subtracted spectrum, plot (c) is the corrected spectrum. Note that the correlation between neighboring bins in the corrected spectrum is of the order of 50\%. Plot (d) is the selection efficiency as a function of the invariant mass. 

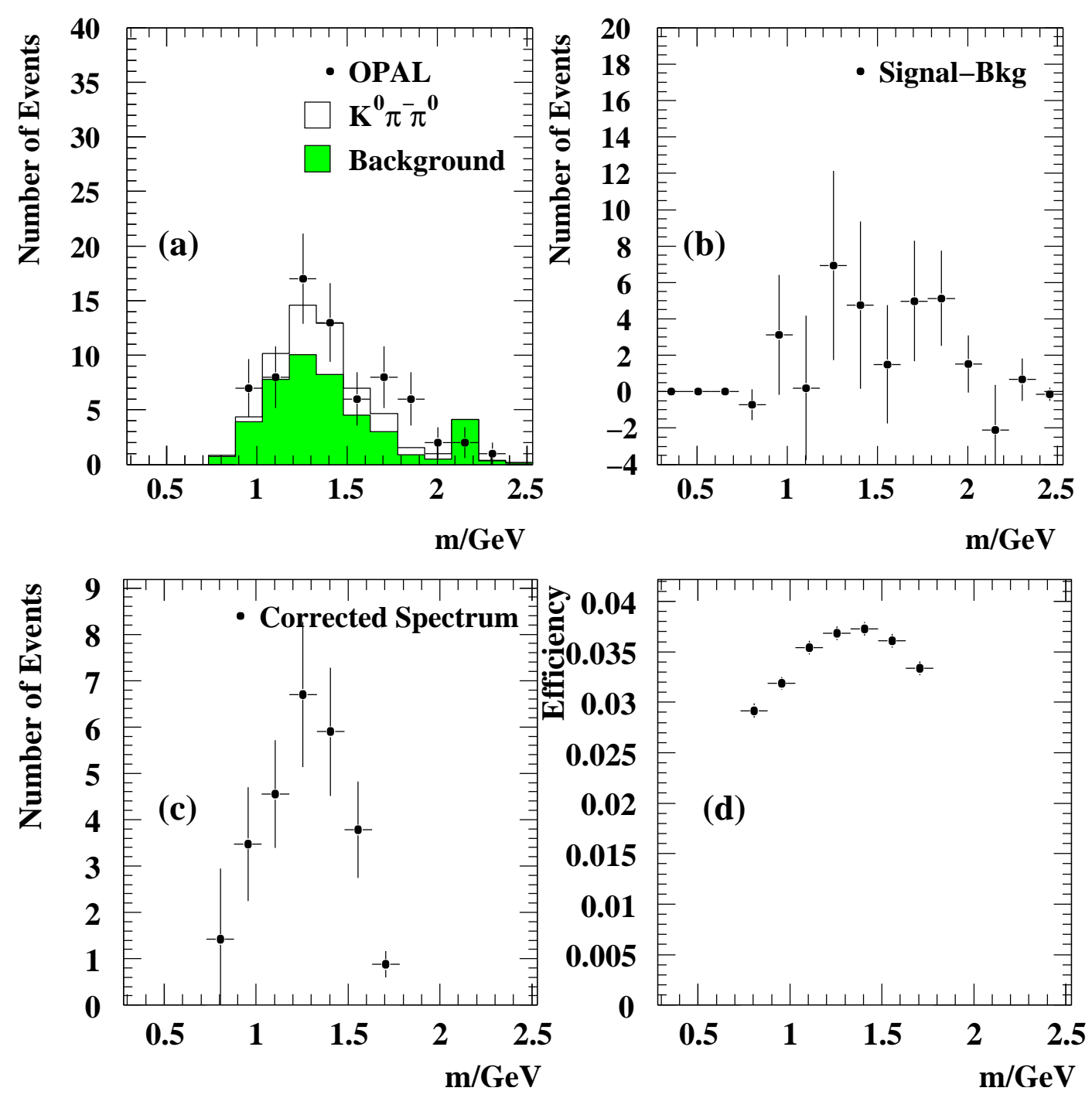

Figure 14: Result of the $\mathrm{K}^{0} \pi^{-} \pi^{0} \nu_{\tau}$ selection. Plot (a) shows the measured invariant mass spectrum. The dots are the data, the open histogram is the Monte Carlo signal and the shaded area is the background. Plot (b) shows the background subtracted spectrum, (c) the corrected spectrum. Note that the correlation between neighboring bins in the corrected spectrum is of the order of 50\%. Plot (d) is the selection efficiency as a function of the invariant mass. 


\section{$4.4(\mathrm{~K} \pi \pi \pi)^{-}$Final States}

The $(\mathrm{K} \pi \pi \pi)^{-}$signal consists of the following final states: $\mathrm{K}^{-} \pi^{+} \pi^{-} \pi^{0} \nu_{\tau}, \mathrm{K}^{0} \pi^{-} \pi^{0} \pi^{0} \nu_{\tau}, \mathrm{K}^{-} \pi^{0} \pi^{0} \pi^{0} \nu_{\tau}$ and $\mathrm{K}^{0} \pi^{-} \pi^{+} \pi^{-} \nu_{\tau}$. From these, only the first one which has the highest branching fraction is investigated.
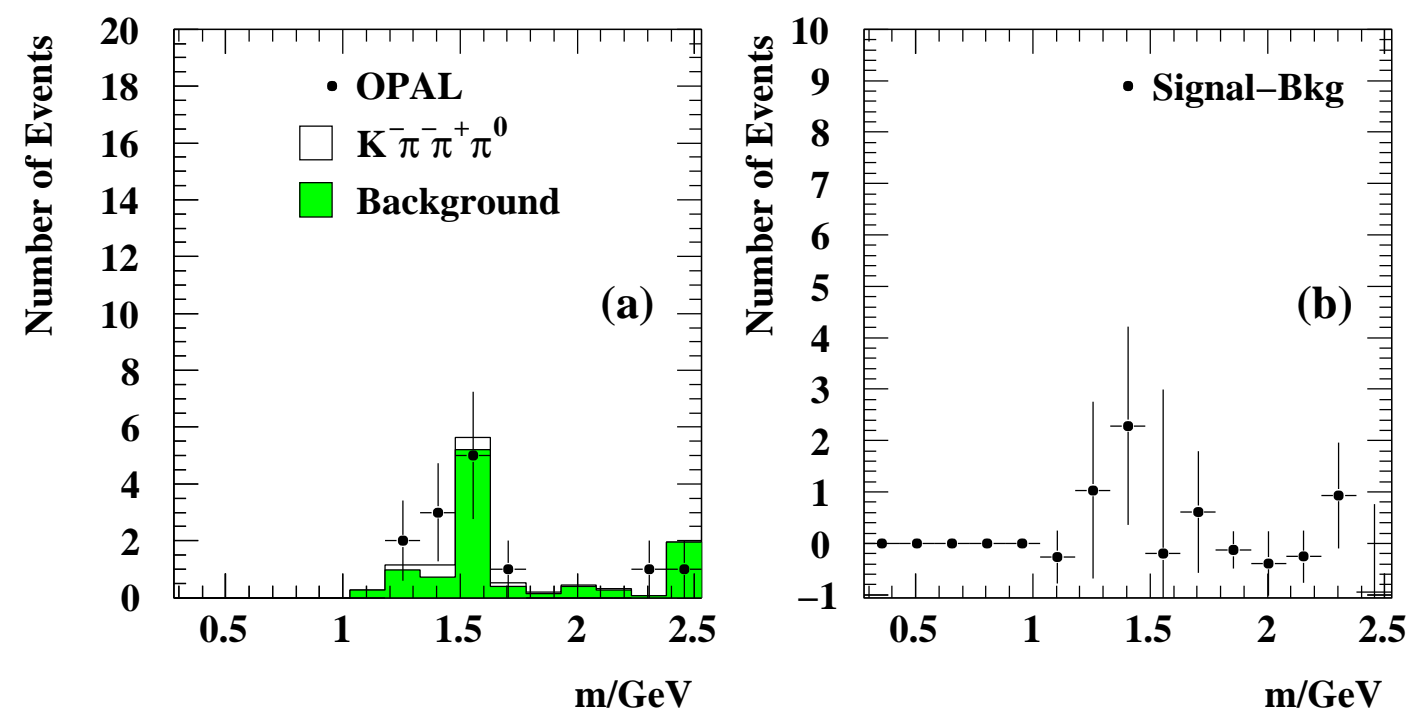

Figure 15: Result of the $\mathrm{K}^{-} \pi^{+} \pi^{-} \pi^{0} \nu_{\tau}$ selection. Plot (a) shows the measured invariant mass spectrum. The dots are the data, the open histogram is the Monte Carlo signal and the shaded area is the background. Plot (b) shows the background subtracted spectrum.

The same procedure as for the $\mathrm{K}^{-} \pi^{+} \pi^{-} \nu_{\tau}$ channel is used. In addition, one identified $\pi^{0}$ meson with an energy of more than $2 \mathrm{GeV}$ is required. The invariant mass spectrum can be seen in Figure 15. The mass resolution in this channel is approximately $60 \mathrm{MeV}$. From this selection, 14 events are seen in the data with a contribution of 10 events from background. The selection efficiency is of the order of $1 \%$. The main background contribution comes from $\tau^{-} \rightarrow \mathrm{K}^{-} \pi^{+} \pi^{-} \nu_{\tau}$ decays, where one fake neutral pion was identified.

Since the number of signal events in this final state is not significantly different from zero, this channel is not considered any further in this analysis. For the spectral function, the Monte Carlo prediction has been used instead.

\section{Results}

\subsection{Branching Fractions}

The measured data used in the spectral function analysis allows the determination of competitive branching fractions for the channels $\tau^{-} \rightarrow \mathrm{K}^{-} \pi^{0} \nu_{\tau}$ and $\tau^{-} \rightarrow \mathrm{K}^{-} \pi^{+} \pi^{-} \nu_{\tau}$. The branching fractions are determined in a simultaneous $\chi^{2}$ fit, taking all measured final states into account. The fit function is

$$
N_{i}=N_{i}^{\mathrm{non}-\tau}+\left(1-f_{\mathrm{bkg}}^{\mathrm{non}-\tau}\right) \cdot N^{\tau} \sum_{j} \varepsilon_{i j} B_{j} F_{j}^{\mathrm{Bias}}
$$

where $i$ is the signal channel under consideration and index $j$ runs over all $\tau$ decay channels. $N_{i}$ is the number of decays observed in the data, i.e. the number of $\tau$ cones passing the cuts. The 
number of decays from the non- $\tau$ background is denoted by $N_{i}^{\mathrm{non}-\tau}, f_{\mathrm{bkg}}^{\mathrm{non}-\tau}$ is the fraction of non$\tau$ background, $N^{\tau}$ is the number of $\tau$ events, $\varepsilon_{i j}$ the efficiency matrix, $F_{j}^{\text {Bias }}$ the bias factor for the respective channel due to $\tau$ preselection cuts and $B_{j}$ are the fitted branching fractions. The branching fractions for the two signal channels were allowed to vary freely, the branching fractions of all other $\tau$ decay channels contributing to the background prediction were allowed to vary only within their PDG errors [14]. The selection quantities used in the fit together with the contributions from the background channels and their branching fractions used in the Monte Carlo simulation are shown in Table 2. From the fit, the following results are obtained:

$$
\begin{aligned}
B\left(\tau^{-} \rightarrow \mathrm{K}^{-} \pi^{0} \nu_{\tau}\right) & =\left(0.471 \pm 0.064_{\text {stat }} \pm 0.022_{\text {sys }}\right) \% \\
B\left(\tau^{-} \rightarrow \mathrm{K}^{-} \pi^{+} \pi^{-}\left(0 \pi^{0}, \text { ex. } \mathrm{K}^{0}\right) \nu_{\tau}\right) & =\left(0.415 \pm 0.059_{\text {stat }} \pm 0.031_{\text {sys }}\right) \%
\end{aligned}
$$

where the possibility that the $\pi^{+} \pi^{-}$pair in the $\mathrm{K}^{-} \pi^{+} \pi^{-} \nu_{\tau}$ final state comes from a $\mathrm{K}_{\mathrm{S}}^{0}$ has been excluded. For the estimation of the systematic uncertainty, the following sources are considered. They are summarized in Table 3. The total systematic uncertainty is obtained by adding the individual contributions in quadrature.

- Energy loss measurement $\left(\Delta_{\mathrm{d} E / \mathrm{d} x}\right)$ :

In the selection, the specific energy loss $\mathrm{d} E / \mathrm{d} x$ is used to separate pions from kaons. Cuts on corresponding weights are applied which are calculated from the pull distribution (see Figure 3). A possible shift in this quantity can lead to a systematic misidentification of tracks. The pull distribution is therefore shifted within the error on its mean and the selection procedure is repeated. The difference between the branching fractions obtained with and without the shift applied is the systematic uncertainty.

- Energy scale in $\pi^{0}$ reconstruction $\left(\Delta_{E}\right)$ :

The energy resolution can be tested by measuring the invariant two-photon mass from $\pi^{0}$ decays. A systematic shift in the observed mass in the data compared to the detector simulation can be translated into a scale factor for the reconstructed photon energies. Deviations of $(0.5 \pm 0.9) \mathrm{MeV}$ from the nominal $\pi^{0}$ mass have been observed [7], corresponding to a scale factor of $1.004 \pm 0.007$. The energies of the reconstructed photons in the Monte Carlo samples were therefore varied by $\pm 0.7 \%$. The difference between the branching fractions obtained with and without the variation applied is the systematic uncertainty.

- Momentum scale $\left(\Delta_{p}\right)$ :

The systematic uncertainty connected with the momentum scale was tested using $\mathrm{Z}^{0} \rightarrow \mu^{-} \mu^{+}$ events [7]. The difference in momentum resolution between data and Monte Carlo as a function of $\cos \theta$ was studied. To assess the systematic uncertainty $\Delta_{p}$ in hadronic $\tau$ decays, all particle momenta in the Monte Carlo were varied accordingly. The difference in the result with and without this variation is quoted as a systematic uncertainty.

- Monte Carlo statistics $\left(\Delta_{\mathrm{MC}}\right)$ :

The precision of the background prediction depends on the Monte Carlo statistics used in the selection procedure. Therefore, the number of background events selected is varied randomly within its statistical uncertainty. The observed spread in the branching fraction due to this variation is quoted as a systematic uncertainty.

- Bias factor $\left(\Delta_{F^{\text {Bias }}}\right)$ :

The bias factors determined from the Monte Carlo are varied by their uncertainty and the branching fractions are then refitted. The observed spread due to this variation contributes to the total systematic uncertainty. 


\begin{tabular}{|c|c|c|c|}
\hline \multicolumn{2}{|l|}{$\tau^{-} \rightarrow \mathrm{K}^{-} \pi^{0} \nu_{\tau}$} & & \\
\hline \multirow{3}{*}{$\begin{array}{l}\text { No. of Events } \\
\text { Selection Efficiency /\% } \\
\text { Preselection Bias Factor } \\
\text { Non- } \tau \text { Background Fraction } \\
\tau \text { Background Fraction } \\
\qquad \begin{array}{r}\pi^{-} \pi^{0} \nu_{\tau} \\
\mathrm{K}^{-} \mathrm{K}^{0} \pi^{0} \nu_{\tau} \\
\mathrm{K}^{-} \nu_{\tau} \\
\mathrm{K}^{-} \mathrm{K}^{0} \nu_{\tau} \\
\pi^{-} \pi^{0} \pi^{0} \nu_{\tau} \\
\mathrm{K}^{-} \pi^{0} \pi^{0} \nu_{\tau} \\
\mathrm{K}^{-} \pi^{0} \pi^{0} \pi^{0} \nu_{\tau} \\
\text { other }\end{array}\end{array}$} & $\begin{array}{c}360 \\
8.42 \pm 0.17 \\
1.016 \pm 0.011 \\
0.006 \pm 0.004 \\
0.540 \pm 0.027\end{array}$ & & \\
\hline & $\begin{array}{c}13.5 \% \\
9.9 \% \\
8.1 \% \\
7.3 \% \\
6.2 \% \\
5.0 \% \\
2.6 \% \\
1.4 \%\end{array}$ & $\begin{array}{lll}0.051 & \pm & 0.005 \\
6.0 & \pm & 0.3 \\
1.25 & \pm & 0.06 \\
4.5 & \pm & 0.2 \\
0.07 & \pm & 0.01 \\
9.6 & \pm & 0.5 \\
8.9 & \pm & 0.6\end{array}$ & 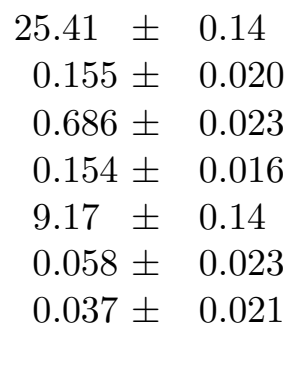 \\
\hline & Bkg. Fraction & Efficiency /\% & $B^{\mathrm{PDG}} / \%$ \\
\hline
\end{tabular}

\begin{tabular}{|c|c|c|c|}
\hline \multicolumn{2}{|c|}{$\tau^{-} \rightarrow \mathrm{K}^{-} \pi^{+} \pi^{-} \nu_{\tau}$} & & \\
\hline $\begin{array}{l}\text { No. of Events } \\
\text { Selection Efficiency/\% } \\
\text { Preselection Bias Factor } \\
\text { Non- } \tau \text { Background Fraction } \\
\tau \text { Background Fraction }\end{array}$ & $\begin{array}{c}269 \\
6.59 \pm 0.06 \\
0.953 \pm 0.013 \\
0.007 \pm 0.006 \\
0.631 \pm 0.044\end{array}$ & & \\
\hline $\begin{array}{r}\pi^{-} \pi^{+} \pi^{-} \nu_{\tau} \\
\pi^{-} \mathrm{K}^{-} \mathrm{K}^{+} \nu_{\tau} \\
\pi^{-} \pi^{+} \pi^{-} \pi^{0} \nu_{\tau} \\
\mathrm{K}^{-} \pi^{+} \pi^{-} \pi^{0} \nu_{\tau} \\
\text { other }\end{array}$ & $\begin{array}{c}21.6 \% \\
10.3 \% \\
8.1 \% \\
6.7 \% \\
16.4 \%\end{array}$ & $\begin{array}{lll}0.15 & \pm & 0.02 \\
3.9 & \pm & 0.2 \\
0.5 & \pm & 0.1 \\
2.7 & \pm & 0.2\end{array}$ & $\begin{array}{ll}9.22 \pm & 0.10 \\
0.161 \pm & 0.019 \\
4.24 \pm & 0.10 \\
0.064 \pm & 0.024\end{array}$ \\
\hline & Bkg. Fraction & Efficiency /\% & $B^{\mathrm{PDG}} / \%$ \\
\hline
\end{tabular}

Table 2: Quantities used in the fit for the branching fractions. The errors quoted for efficiency, bias factor and background fractions are from Monte Carlo statistics only. The last column contains the branching fractions for the background channels used in the Monte Carlo simulation [14].

\begin{tabular}{|l|c|c|}
\cline { 2 - 3 } \multicolumn{1}{c|}{} & $\tau^{-} \rightarrow \mathrm{K}^{-} \pi^{0} \nu_{\tau}$ & $\tau^{-} \rightarrow \mathrm{K}^{-} \pi^{+} \pi^{-} \nu_{\tau}$ \\
\hline Energy Loss Measurement $(\mathrm{d} E / \mathrm{d} x)$ & 0.012 & 0.019 \\
Energy Scale & 0.010 & 0.011 \\
Momentum Scale & 0.003 & 0.003 \\
MC Statistics & 0.014 & 0.021 \\
Bias Factor $\left(F^{\text {Bias }}\right)$ & 0.004 & 0.005 \\
\hline \hline Total & 0.022 & 0.031 \\
\hline
\end{tabular}

Table 3: Individual contributions to the systematic uncertainty of the branching fraction measurements as explained in the text. 


\subsection{Improved Averages for $B\left(\tau^{-} \rightarrow \mathrm{K}^{-} \pi^{0} \nu_{\tau}\right)$ and $B\left(\tau^{-} \rightarrow \mathrm{K}^{-} \pi^{+} \pi^{-} \nu_{\tau}\right)$}

For the determination of the spectral function and its moments described below, new average values for the branching fractions of the decays $\tau^{-} \rightarrow \mathrm{K}^{-} \pi^{0} \nu_{\tau}$ and $\tau^{-} \rightarrow \mathrm{K}^{-} \pi^{+} \pi^{-} \nu_{\tau}$ are determined. In addition to the results obtained here, the same measurements are used as inputs for the calculation as in [14]. For the channel $\tau^{-} \rightarrow \mathrm{K}^{-} \pi^{+} \pi^{-} \nu_{\tau}$, the previous result from OPAL [32] was replaced by the measurement obtained in this analysis. Also, the CLEO result was updated using [33]. The new averages are:

$$
\begin{aligned}
B_{\mathrm{av}}\left(\tau^{-} \rightarrow \mathrm{K}^{-} \pi^{0} \nu_{\tau}\right) & =(0.453 \pm 0.030) \% \\
B_{\mathrm{av}}\left(\tau^{-} \rightarrow \mathrm{K}^{-} \pi^{+} \pi^{-} \nu_{\tau}\right) & =(0.330 \pm 0.028) \%
\end{aligned}
$$

The measurements used, together with the averages given in [14] and the improved values for the branching fractions obtained here are displayed in Figure 16]

The branching fraction obtained from this analysis for the $\mathrm{K}^{-} \pi^{0} \nu_{\tau}$ channel is consistent with the previous measurements within the errors quoted. The value obtained for the $\mathrm{K}^{-} \pi^{+} \pi^{-} \nu_{\tau}$ channel is consistent with the new measurement from CLEO and the theoretical prediction in [22]. The ALEPH [34] result differs from these values by roughly $2.5 \sigma$.
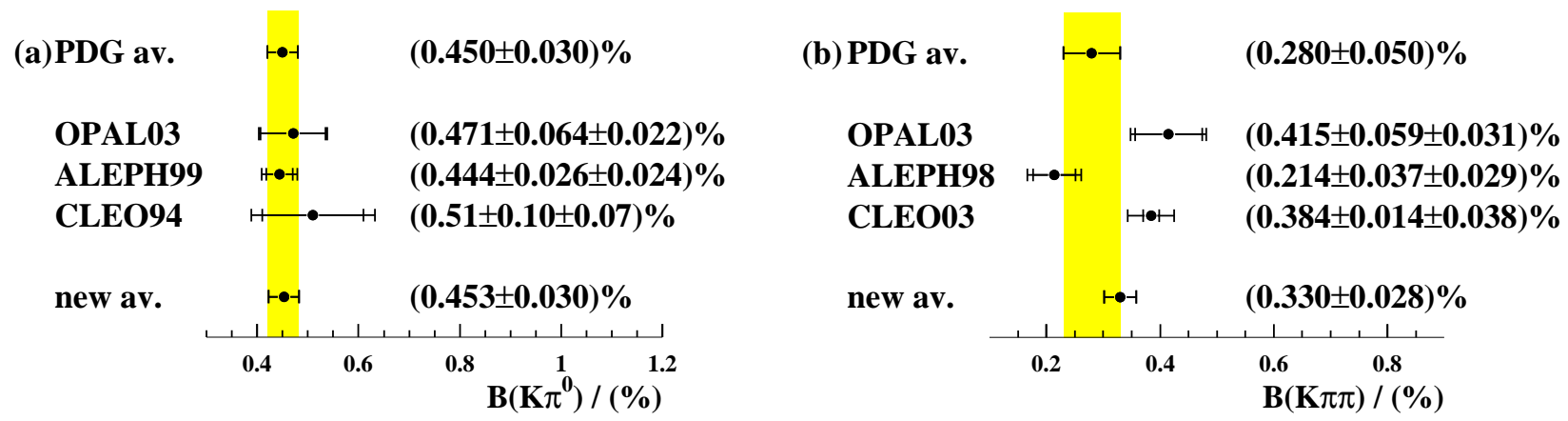

Figure 16: New averages for the branching fractions for $\tau^{-} \rightarrow \mathrm{K}^{-} \pi^{0} \nu_{\tau}$ (a) and $\tau^{-} \rightarrow \mathrm{K}^{-} \pi^{+} \pi^{-} \nu_{\tau}$ (b). The previous world average as given by the PDG is indicated by the shaded band. The measurements used in the calculation of the weighted mean and the new averages are shown. OPAL03 denotes the values for the branching fractions obtained from this analysis.

\subsection{The Strangeness Spectral Function}

The hadronic decay of the $\tau$ lepton is commonly written in terms of the so-called spectral functions $v_{1}(s), a_{0 / 1}(s)$ for the non-strange part and $v_{0 / 1}^{\mathrm{S}}(s)$ and $a_{0 / 1}^{\mathrm{S}}(s)$ for the strange part. The functions $v$ and $a$ are the vector $(\mathrm{V})$ and the axialvector (A) contributions, respectively, while the subscript denotes the angular momentum $J$. The variable $s$ is the invariant mass squared of the hadronic system. The spectral function is experimentally determined by measuring the invariant mass spectra of the given hadronic modes and normalizing them to their respective branching fractions. The contributions to the total strangeness spectral function then read:

$$
v_{1}^{\mathrm{S}}(s) / a_{1}^{\mathrm{S}}(s)=\frac{m_{\tau}^{2}}{6\left|V_{\mathrm{us}}\right|^{2} S_{\mathrm{ew}}}\left(1-\frac{s}{m_{\tau}^{2}}\right)^{-2}\left(1+\frac{2 s}{m_{\tau}^{2}}\right)^{-1} \frac{B\left(\tau \rightarrow(V / A)^{(S=-1, J=1)} \nu_{\tau}\right)}{B\left(\tau \rightarrow e^{-} \bar{\nu}_{e} \nu_{\tau}\right)} \frac{1}{N_{\mathrm{V} / \mathrm{A}}} \frac{\mathrm{d} N_{\mathrm{V} / \mathrm{A}}}{\mathrm{d} s}
$$

and

$$
v_{0}^{\mathrm{S}}(s) / a_{0}^{\mathrm{S}}(s)=\frac{m_{\tau}^{2}}{6\left|V_{\mathrm{us}}\right|^{2} S_{\mathrm{ew}}}\left(1-\frac{s}{m_{\tau}^{2}}\right)^{-2} \frac{B\left(\tau \rightarrow(V / A)^{(S=-1, J=0)} \nu_{\tau}\right)}{B\left(\tau \rightarrow e^{-} \bar{\nu}_{e} \nu_{\tau}\right)} \frac{1}{N_{\mathrm{V} / \mathrm{A}}} \frac{\mathrm{d} N_{\mathrm{V} / \mathrm{A}}}{\mathrm{d} s}
$$


where $\left|V_{\mathrm{us}}\right|=0.2196 \pm 0.0023[14]$ is the CKM weak mixing matrix element, $m_{\tau}=\left(1776.9_{-0.27}^{+0.31}\right) \mathrm{MeV}$ [35] and $S_{\text {ew }}=1.0194 \pm 0.0040$ [36] is an electroweak correction factor. The total strangeness spectral function $(v+a)$ is then obtained by adding the individual contributions. To disentangle the vector and the axialvector contributions for the spin-1 part, a detailed analysis of the resonance structure of the measured spectra is necessary which is not done here due to the limited statistics. The kaon pole contributes to the pseudoscalar spin-0 part.

Summing the individual contributions (see Equations 3 and [4), the strangeness spectral function is obtained. The Monte Carlo prediction of the total strangeness spectral function as a function of the invariant mass squared is displayed in Figure [17. The improved version of the $\tau$ Monte Carlo as explained in Section 2.3 has been used here. The branching fractions as given in Table 1 have been used to weight the individual mass spectra. For illustration purposes the spectral function is shown using two different binnings. A non-equidistant binning is chosen which corresponds to a bin width of $50 \mathrm{MeV}$ and $150 \mathrm{MeV}$ in the invariant mass, respectively. The errors given here are from Monte Carlo statistics only.

The spectral function obtained from the data is displayed in Figure 18. For $\tau^{-} \rightarrow \mathrm{K}^{-} \pi^{0} \nu_{\tau}$ and $\tau^{-} \rightarrow \mathrm{K}^{-} \pi^{+} \pi^{-} \nu_{\tau}$, the new average branching fractions and their respective errors as given in Section 5.2 are used. The binning chosen in this plot corresponds to a bin width of $150 \mathrm{MeV}$ in the invariant mass and is governed by the mass resolution of the $\mathrm{K}^{0} \pi^{-} \pi^{0} \nu_{\tau}$ final state. The dots with error bars represent the inclusive spectrum. The inner error bars are the statistical uncertainties. They include the uncertainty on the efficiency and on Monte Carlo statistics. The total error is calculated by adding up the statistical and systematic uncertainties (explained in the next section) in quadrature. The numerical values are given in Table 4. The systematic uncertainty is dominated by the uncertainty on the $\tau$ branching fractions.

For the $(\mathrm{K} \pi)^{-}$final state both $\tau$ decay channels $\mathrm{K}^{0} \pi^{-} \nu_{\tau}$ and $\mathrm{K}^{-} \pi^{0} \nu_{\tau}$ are measured. The channel $\mathrm{K}^{-} \eta \nu_{\tau}$ which also contributes to the two meson final state is taken from Monte Carlo. For the $(\mathrm{K} \pi \pi)^{-}$final state the spectra $\mathrm{K}^{0} \pi^{-} \pi^{0} \nu_{\tau}$ and $\mathrm{K}^{-} \pi^{+} \pi^{-} \nu_{\tau}$ are measured. The contribution from the decay $\mathrm{K}^{-} \pi^{0} \pi^{0} \nu_{\tau}$ is added from Monte Carlo as well as the $\mathrm{K}^{-} \eta \pi^{0} \nu_{\tau}$ channel which also contributes to the three meson final states. For the $(\mathrm{K} \pi \pi \pi)^{-}$spectrum, which consists of the channels

$\mathrm{K}^{-} \pi^{+} \pi^{-} \pi^{0} \nu_{\tau}, \mathrm{K}^{0} \pi^{-} \pi^{0} \pi^{0} \nu_{\tau}, \mathrm{K}^{-} \pi^{0} \pi^{0} \pi^{0} \nu_{\tau}$ and $\mathrm{K}^{0} \pi^{-} \pi^{+} \pi^{-} \nu_{\tau}$, the prediction from the Monte Carlo is taken.

\subsection{Systematic Uncertainties on the Spectral Function}

The sources for possible systematic uncertainties listed below have been considered. Since the individual contributions are different for the different final states, the error is given for each bin in $s$ separately in Table 4 .

- Energy loss measurement $\left(\Delta_{\mathrm{d} E / \mathrm{d} x}\right)$ :

The systematic variation is described in Section 5.1. In addition, the momentum dependence of this variation has been investigated. No significant influence has been found.

- Energy scale in $\pi^{0}$ reconstruction $\left(\Delta_{\mathrm{E}}\right)$ :

The systematic variation is described in Section 5.1

- Momentum scale $\left(\Delta_{\mathrm{p}}\right)$ :

The systematic variation is described in Section 5.1 .

- PDG errors on branching fractions $\left(\Delta_{\mathrm{B}}\right)$ :

The dominant contribution to the systematic uncertainty comes from the uncertainty in the 
$\tau$ branching fractions (see Table 1). The branching fractions are varied randomly and the difference in the result is quoted as systematic uncertainty. The channels which populate the region of high $s$ have branching fractions with relative errors close to $100 \%$ leading to large uncertainty in the spectral function itself. This also covers the uncertainties on the shape.

- $\mathrm{K}_{\mathrm{S}}^{0}$ identification $\left(\Delta_{\mathrm{K}_{\mathrm{S}}^{0}}\right)$ :

A possible origin for systematic effects in the $\mathrm{K}_{\mathrm{S}}^{0}$ identification is the estimation of the background using Monte Carlo. In particular the number of photon conversions found in $\tau$ lepton decays is not perfectly modeled. The cut on the $\chi^{2}$ probability of the $2 \mathrm{C}$ constrained fit to $\mathrm{K}_{\mathrm{S}}^{0}$ (see Section [3.3) has been varied from $10^{-5}$ to 0.01 to estimate a possible systematic effect. This cut reduces the number photon conversions in the sample by one order of magnitude.

- Mass correction procedure $\left(\Delta_{\text {mcorr }}\right)$ :

In order to assess the systematic uncertainty associated with the mass correction procedure, two possible sources have to be considered. The effect due to different input mass spectra and the effect due to the choice of the number of iterations. They have been studied using high statistics event samples. The systematic deviations using either flat, phase space, or resonance shaped input spectra (with an optimized number of iterations for each scenario) are about $5 \%$ in each mass bin. The deviations are largest when a flat input distribution is assumed. Because the true mass spectrum is certainly not flat, we consider $5 \%$ to be a conservative estimate for $\Delta_{\text {mcorr }}$. The effect of the number of iterations was estimated using a phase space distribution for input spectrum and performing one additional iteration step. The change was found to be negligible compared to the effect of varying the input distribution.

\begin{tabular}{|c||c|c|c|c|c|c||cc||c|}
\hline$(s$-range $) / \mathrm{GeV}^{2}$ & $\Delta_{\mathrm{B}}$ & $\Delta_{\mathrm{d} E / \mathrm{d} x}$ & $\Delta_{\mathrm{K}_{\mathrm{S}}^{0}}$ & $\Delta_{\mathrm{E}}$ & $\Delta_{\mathrm{p}}$ & $\Delta_{\text {mcorr }}$ & $\Delta_{\text {sys }}^{\text {tot }}$ & $\Delta_{\text {stat }}$ & $v+a$ \\
\hline \hline$(0.53,0.77)$ & 0.04 & 0.006 & 0.006 & 0.007 & 0.003 & 0.06 & 0.07 & 0.17 & $1.17 \pm 0.18$ \\
$(0.77,1.06)$ & 0.13 & 0.011 & 0.011 & 0.014 & 0.001 & 0.11 & 0.17 & 0.18 & $2.27 \pm 0.25$ \\
$(1.06,1.39)$ & 0.08 & 0.003 & 0.003 & 0.004 & 0.001 & 0.03 & 0.09 & 0.07 & $0.69 \pm 0.11$ \\
$(1.39,1.77)$ & 0.18 & 0.005 & 0.005 & 0.005 & 0.002 & 0.05 & 0.18 & 0.19 & $0.90 \pm 0.26$ \\
$(1.77,2.19)$ & 0.32 & 0.006 & 0.007 & 0.007 & 0.003 & 0.06 & 0.33 & 0.25 & $1.22 \pm 0.41$ \\
$(2.19,2.66)$ & 0.35 & 0.007 & 0.009 & 0.009 & 0.003 & 0.07 & 0.36 & 0.49 & $1.44 \pm 0.61$ \\
$(2.66,3.17)$ & 0.30 & 0.007 & 0.008 & 0.008 & 0.003 & 0.07 & 0.31 & 0.85 & $1.35 \pm 0.90$ \\
\hline
\end{tabular}

Table 4: Result for the strangeness spectral function. The table shows the values of the total strangeness spectral function $(v+a)$ and the statistical and the total systematic uncertainties for every bin in s. The individual contributions to the systematic uncertainty are discussed in the text. The total uncertainty quoted is the quadratic sum of the statistical and systematic uncertainties.

\subsection{The Spectral Moments $R_{\tau, S}^{k l}$}

The moments of the spectral function are defined as:

$$
R_{\tau, S}^{k l}\left(m_{\tau}^{2}\right)=\int_{0}^{m_{\tau}^{2}} \mathrm{~d} s\left(1-\frac{s}{m_{\tau}^{2}}\right)^{k}\left(\frac{s}{m_{\tau}^{2}}\right)^{l} \frac{B\left(\tau \rightarrow(V / A)^{(S=-1, J=0 / 1)} \nu_{\tau}\right)}{B\left(\tau \rightarrow e^{-} \bar{\nu}_{e} \nu_{\tau}\right)} \frac{1}{N_{\mathrm{V} / \mathrm{A}}} \frac{\mathrm{d} N_{\mathrm{V} / \mathrm{A}}}{\mathrm{d} s},
$$

where the sum runs over all final states with open strangeness. The values measured for the moments $k l=(00,10,11,12,13,20,21,30,40)$, their statistical and systematic uncertainties are given in Table [5. The various sources contributing to the systematic uncertainty have been discussed in Section [5.4 The value for $R_{\tau, S}^{00}$ is calculated from the branching fractions only and is 
therefore independent of the measured spectra. In addition, the CKM-weighted difference of the corresponding strange and non-strange moments is given:

$$
\delta R_{\tau}^{k l}=\frac{R_{\tau, n o n-S}^{k l}}{\left|V_{\mathrm{ud}}\right|^{2}}-\frac{R_{\tau, S}^{k l}}{\left|V_{\mathrm{us}}\right|^{2}},
$$

(see Table 5) where $R_{\tau, S}^{k l}$ are the strange moments and $R_{\tau, n o n-S}^{k l}$ is the sum of the vector- and axialvector non-strange moments from [7], using updated branching fractions. The values of the matrix elements used for the non-strange and strange moments are $\left|V_{\mathrm{ud}}\right|=(0.9734 \pm 0.0008)$ and $\left|V_{\mathrm{us}}\right|=(0.2196 \pm 0.0023)$, respectively [14]. Systematic errors which are common to both, strange and non-strange moments, such as the energy scale error and the momentum scale for tracks, are $100 \%$ correlated and are treated accordingly.

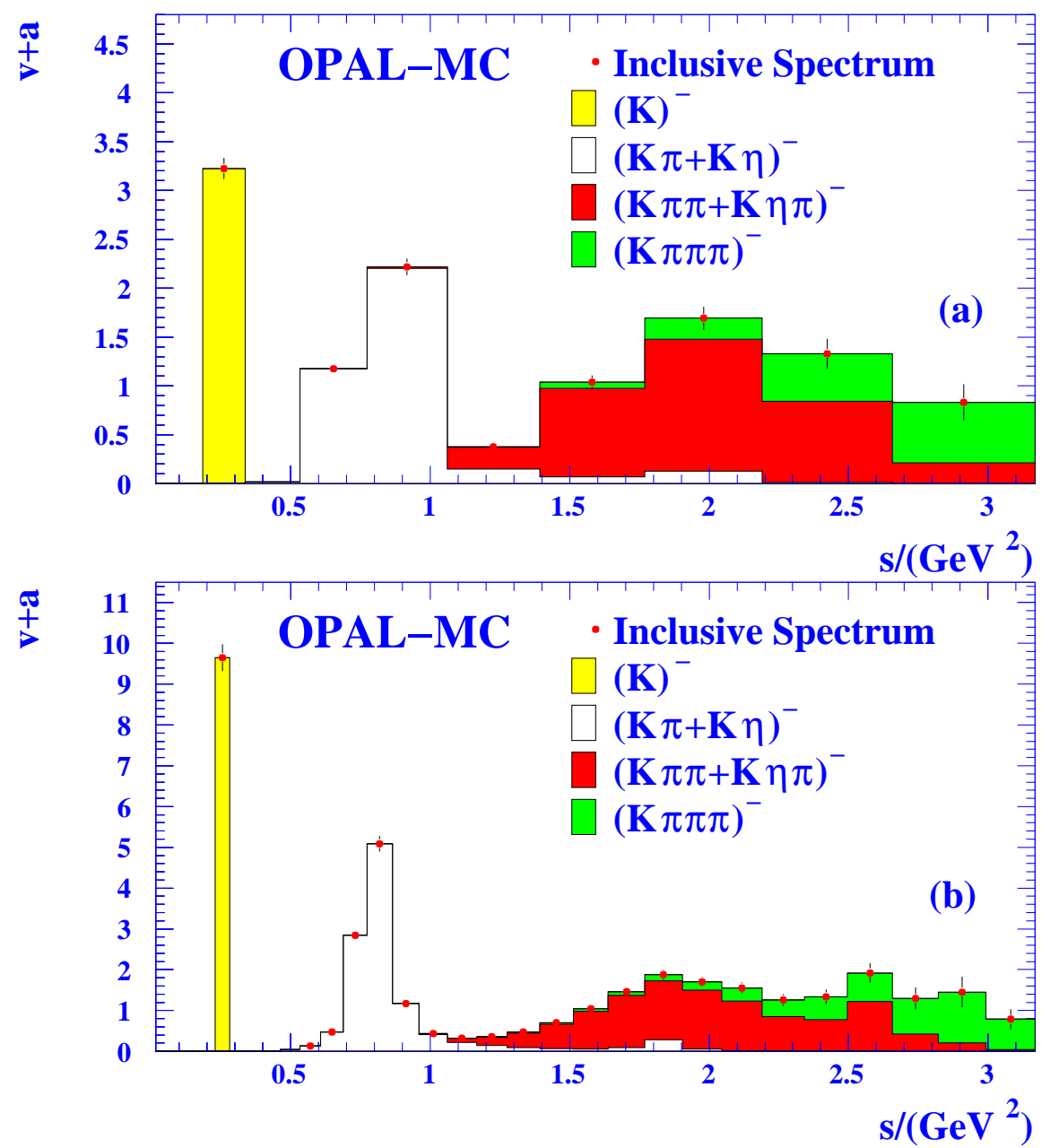

Figure 17: The strangeness spectral function as determined from Monte Carlo. For illustration purposes two different binnings were used - (a) $150 \mathrm{MeV}$ and (b) $50 \mathrm{MeV}$ in mass. The dots represent the inclusive spectrum. The white histogram denotes the contribution from the $(\mathrm{K} \pi)^{-}$and $(\mathrm{K} \eta)^{-}$final states, the dark shaded histogram those from the $(\mathrm{K} \pi \pi)^{-}$and $(\mathrm{K} \eta \pi)^{-}$channels and the light shaded area shows the contribution from $(\mathrm{K} \pi \pi \pi)^{-}$final states. The leftmost bin represents the kaon pole which is the only contribution to the spin-0 part. The errors shown include the statistical uncertainty of the Monte Carlo as well as the uncertainties on the branching fractions. 


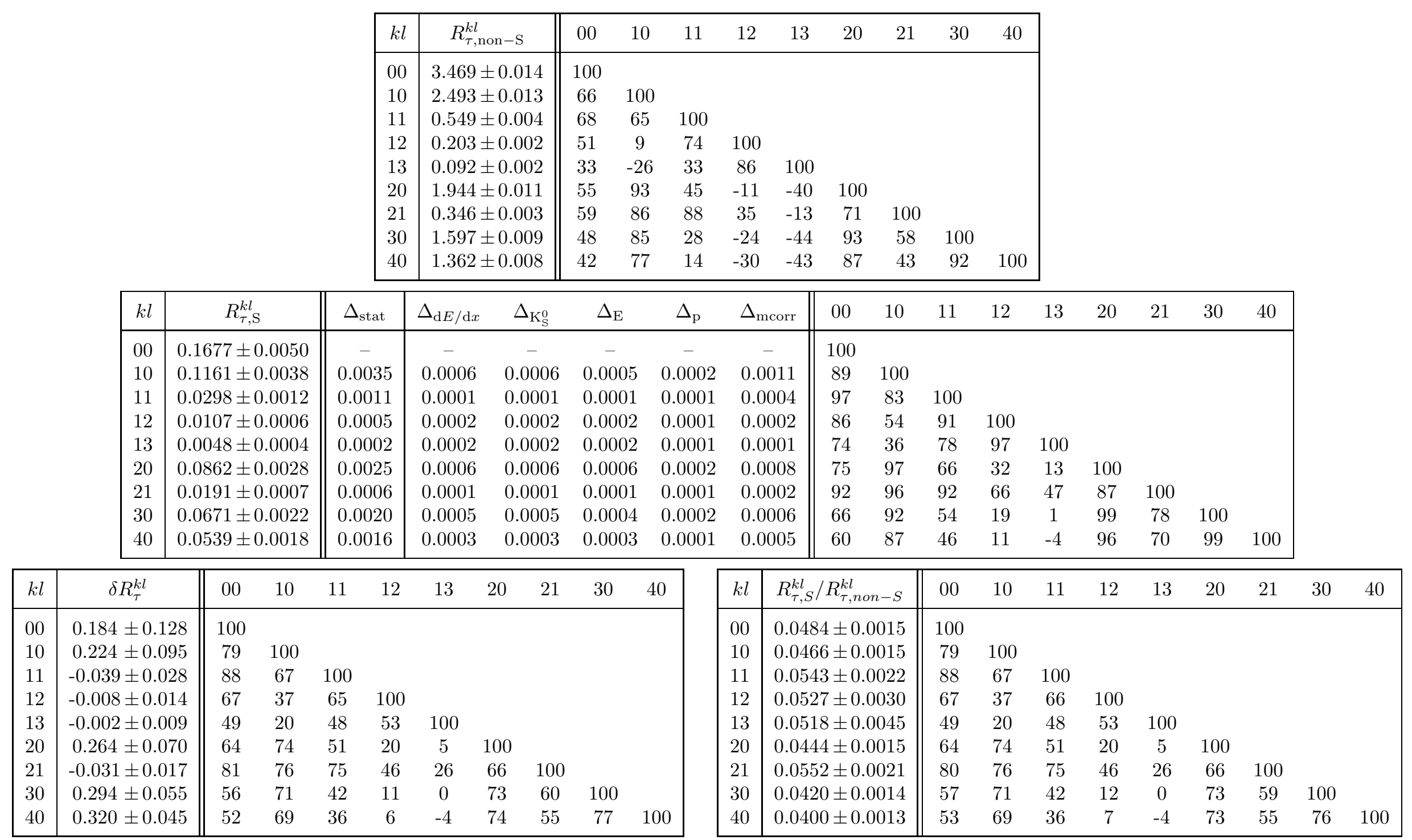

Table 5: The spectral moments for $k l=(00,10,11,12,13,20,21,30,40)$. The tables include the values for the non-strange moments, the strange moments, the weighted difference and the quotient of strange to non-strange moments. The values for the non-strange moments given are calculated from [7] using updated branching fractions. For the strange moments, statistical and systematic uncertainties are listed separately for the individual sources. The statistical uncertainty also contains the uncertainty on the branching fractions. The uncertainty on $R_{\tau}^{00}$ is the total error from the branching fractions as given in [14]. On the right hand side of each table, the correlations are given in percent. 


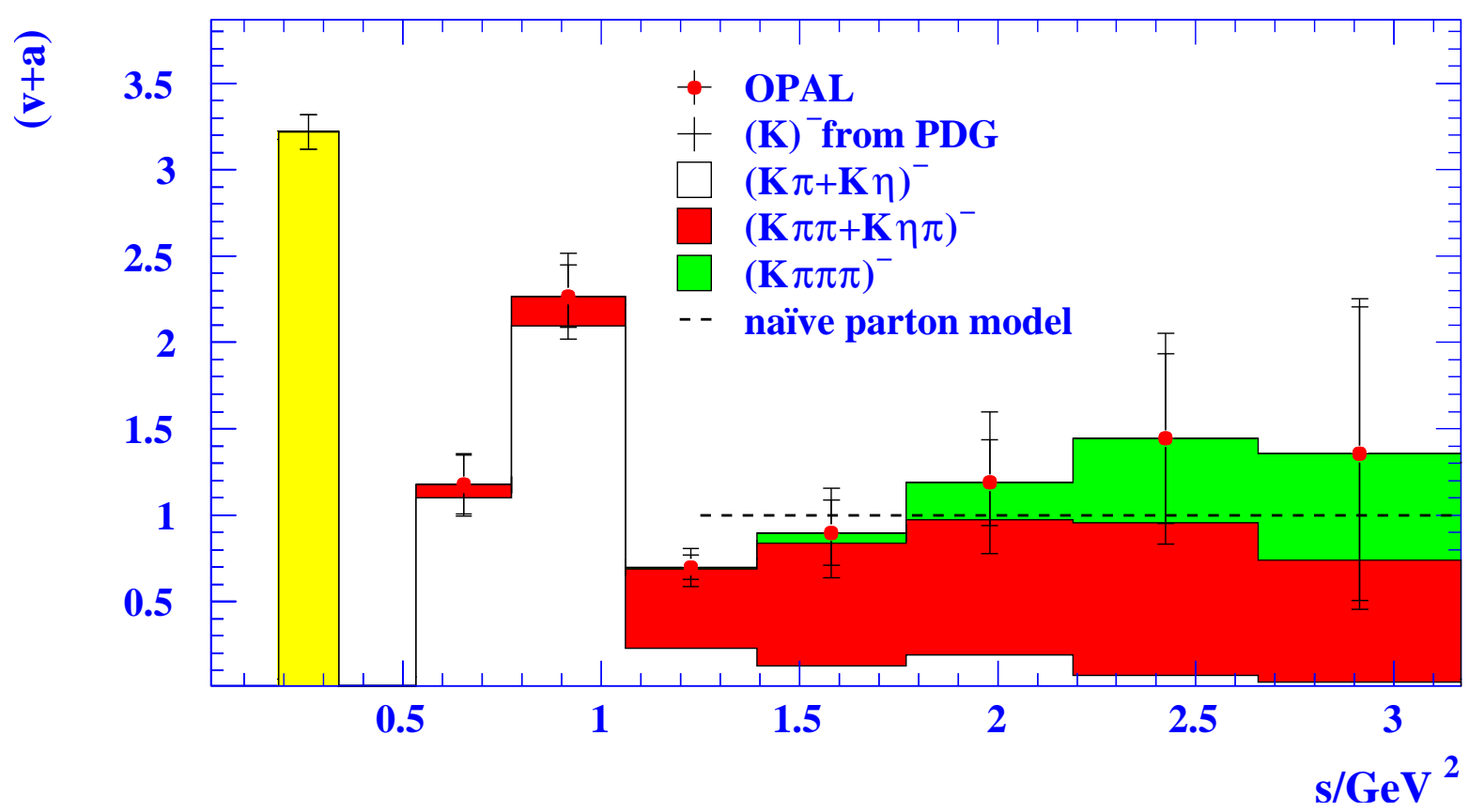

Figure 18: The spectral function from strange $\tau$ lepton decays. The dots show the inclusive spectrum as measured in OPAL. The white histogram shows the exclusive $(\mathrm{K} \pi+\mathrm{K} \eta)^{-}$spectrum, the dark shaded area the $(\mathrm{K} \pi \pi+\mathrm{K} \eta \pi)^{-}$contribution and the light shaded area the contribution from the $(\mathrm{K} \pi \pi \pi)^{-}$final states. The error bars show the statistical uncertainty (inner error bar) and the systematic uncertainty added in quadrature (total error).

\section{Conclusions}

The spectral function in $\tau$ lepton decays into vector and axial vector final states with open strangeness, as well as the spectral moments $R_{\tau, S}^{k l}$, have been measured with the OPAL detector, operating on the $\mathrm{Z}^{0}$ resonance. The selection of the decay channels requires a detailed study of the charged kaon identification using $\mathrm{d} E / \mathrm{d} x$, neutral pion reconstruction in the electromagnetic calorimeter, and identification of $\mathrm{K}_{\mathrm{S}}^{0}$. For the decays $\tau^{-} \rightarrow(\mathrm{K} \pi)^{-} \nu_{\tau},(\mathrm{K} \pi \pi)^{-} \nu_{\tau}$ and $(\mathrm{K} \pi \pi \pi)^{-} \nu_{\tau}, 94 \%$ of all final states have been experimentally accessed; for the remaining $6.6 \%$, as well as for the strange final states including $\eta$ mesons, Monte Carlo simulations have been used. The reconstructed strange final states corrected for resolution effects and detection efficiencies yield the strangeness spectral function of the $\tau$ lepton. The spectral moments $R_{\tau, S}^{k l}$ for $k l=\{10,11,12,13,20,21,30,40\}$, as well as the ratio of strange to non-strange moments, updated from [7], and the weighted differences $\delta R_{\tau}^{k l}$ are given, which are useful quantities for further theoretical analysis. For the decay channels $\tau^{-} \rightarrow \mathrm{K}^{-} \pi^{0} \nu_{\tau}$ and $\tau^{-} \rightarrow \mathrm{K}^{-} \pi^{+} \pi^{-} \nu_{\tau}$ competitive branching fractions are obtained:

$$
\begin{aligned}
B\left(\tau^{-} \rightarrow \mathrm{K}^{-} \pi^{0} \nu_{\tau}\right) & =\left(0.471 \pm 0.064_{\text {stat }} \pm 0.022_{\text {sys }}\right) \% \\
B\left(\tau^{-} \rightarrow \mathrm{K}^{-} \pi^{+} \pi^{-} \nu_{\tau}\right) & =\left(0.415 \pm 0.059_{\text {stat }} \pm 0.031_{\text {sys }}\right) \% .
\end{aligned}
$$


We particularly wish to thank the SL Division for the efficient operation of the LEP accelerator at all energies and for their close cooperation with our experimental group. In addition to the support staff at our own institutions we are pleased to acknowledge the

Department of Energy, USA,

National Science Foundation, USA,

Particle Physics and Astronomy Research Council, UK,

Natural Sciences and Engineering Research Council, Canada,

Israel Science Foundation, administered by the Israel Academy of Science and Humanities,

Benoziyo Center for High Energy Physics,

Japanese Ministry of Education, Culture, Sports, Science and Technology (MEXT) and a grant under the MEXT International Science Research Program,

Japanese Society for the Promotion of Science (JSPS),

German Israeli Bi-national Science Foundation (GIF),

Bundesministerium für Bildung und Forschung, Germany,

National Research Council of Canada,

Hungarian Foundation for Scientific Research, OTKA T-038240, and T-042864,

The NWO/NATO Fund for Scientific Research, the Netherlands.

\section{References}

[1] E. Braaten, S. Narison and A. Pich, Nucl. Phys. B373, (1992) 581.

[2] E. Braaten, Phys. Rev. Lett. 60, (1988) 1606.

[3] E. Braaten, Phys. Rev. D39, (1989) 1458.

[4] S. Narison and A. Pich, Phys. Lett. B211, (1988) 183.

[5] F. Le Diberder and A. Pich, Phys. Lett. B286, (1992) 147.

[6] A. A. Pivovarov, Z. Phys. C 53, (1992) 461.

[7] OPAL Collaboration, K. Ackerstaff et al., Eur. Phys. J. C7, (1999) 571.

[8] ALEPH Collaboration, R. Barate et al., Eur. Phys. J. C4, (1998) 409.

[9] CLEO Collaboration, T. Coan et al., Phys. Lett. B356, (1995) 580.

[10] See for example A. Pich, 'QCD predictions for the tau hadronic width: Determination of $\alpha_{\mathrm{S}}\left(m_{\tau}\right)$ ' in Proceedings of the 2nd Workshop on Tau Lepton Physics, edited by K. K. Ghan, Columbus, Ohio, 1992.

[11] K. G. Chetyrkin, J. H. Kuhn and A. A. Pivovarov, Nucl. Phys. B 533, (1998) 473.

[12] A. Pich and J. Prades, JHEP 9910, (1999) 004.

[13] K. Maltman, eConf C0209101, (2002) WE05.

[14] K. Hagiwara et al., Phys. Rev. D 66, (2002) 010001 (URL: http://pdg.lbl.gov/2003/s035.pdf).

[15] OPAL Collaboration, G. Abbiendi et al., Eur. Phys. J. C13, (2000) 197.

[16] OPAL Collaboration, R. Akers et al., Phys. Lett. B328, (1994) 207. 
[17] OPAL Collaboration, K. Ahmet et al., Nucl. Instr. and Meth. A 305, (1991) 275.

[18] P.P. Allport et al., Nucl. Instr. and Meth. A 324, (1993) 34; P.P. Allport et al., Nucl. Instr. and Meth. A 346, (1994) 476.

[19] S. Jadach, B.F.L. Ward, and Z. Wa̧s, Comp. Phys. Comm. 79, (1994) 503.

[20] S. Jadach, Z. Wąs, R. Decker, and J.H. Kühn, Comp. Phys. Comm. 76, (1993) 361.

[21] M. Finkemeier and E. Mirkes, Z. Phys. C 69, (1996) 243.

[22] M. Finkemeier, J. H. Kühn and E. Mirkes, Nucl. Phys. Proc. Suppl. 55C, (1997) 169.

[23] J. Allison et al., Nucl. Instrum. Methods A317, (1992) 47.

[24] T. Sjöstrand, Comp. Phys. Comm. 82, (1994) 74.

[25] S. Jadach, W. Placzek and B. F. Ward, Phys. Lett. B 390, (1997) 298.

[26] R. Engel, and J. Ranft Phys. Rev. D54, (1996) 4244.

[27] A. Buijs, W. G. Langeveld, M. H. Lehto and D. J. Miller, Comput. Phys. Commun. 79, (1994) 523.

[28] R. Battacharya, J. Smith, and G. Grammer, Phys. Rev. D15, (1977) 3267.

[29] J. Smith, J.A.M. Vermaseren, and G. Grammer, Phys. Rev. D15, (1977) 3280.

[30] M. Hauschild et al., Nucl. Inst. Meth. A314, (1992) 74.

[31] S. Bethke, Z. Kunszt, D.E. Soper and W.J. Stirling: Nucl. Phys. B370, (1992) 310, erratum ibid. B523, (1998) 681.

[32] OPAL Collaboration ,G. Abbiendi et al., Eur. Phys. J. C 13, (2000) 197.

[33] CLEO Collaboration, R. A. Briere et al., Phys. Rev. Lett. 90, (2003) 181802.

[34] ALEPH Collaboration, R. Barate et al., Eur. Phys. J. C 1, (1998) 65.

[35] BES Collaboration, J.Z. Bai et al., Phys. Rev. D53, (1996) 20.

[36] W.J. Marciano and A. Sirlin, Phys. Rev. Lett. 61, (1988) 1815. 